\title{
Klima der Region - Zustand, bisherige Entwicklung und mögliche Änderungen bis 2100
}

Insa Meinke, Diana Rechid, Birger Tinz, Moritz Maneke, Christiana Lefebvre, Elke Isokeit

2.1 Einführung - 16

$2.2 \quad$ Klimazustand - 16

2.2.1 Wind -17

2.2.2 Luftemperatur - 18

2.2.3 Niederschlag - 20

2.2.4 Sonnenscheindauer - 21

2.3 Bisherige klimatische Entwicklung in der Region - 21

2.3.1 Die atmosphärische Zirkulation - 22

2.3.2 Wind -22

2.3.3 Lufttemperatur - 23

2.3.4 Niederschlag -25

2.4 Mögliche Änderungen des Klimas im 21. Jahrhundert - 27

2.4.1 Einleitung: Klimaprojektionen für das 21. Jahrhundert - 27

2.4.2 Projizierte Klimaänderungen in der Metropolregion Hamburg im 21. Jahrhundert - 28

2.5 Zusammenfassung und Ausblick - 34

Literatur - 34 


\subsection{Einführung}

Das Fachwissen zum Klima in der Metropolregion Hamburg (MRH) und seinen Änderungen wurde bis 2008 ausführlich im „Klimabericht für die Metropolregion Hamburg“ dokumentiert (Rosenhagen und Schatzmann 2011; Daschkeit 2011). Im Jahr 2013 haben die Leitautoren im Rahmen einer Aktualisierung des Klimaberichtes bzgl. dieses Themenfeldes auf das BMBFProjekt KLIMZUG-NORD und das Hamburger Exzellenzcluster CliSAP sowie erste daraus entstandene Arbeiten verwiesen (Rosenhagen 2013), die sich vielfach auf das Stadtklima Hamburgs beziehen (vgl. Kap. 3). Inzwischen stehen für weitere Regionen in Norddeutschland Ergebnisse aus verschiedenen Forschungsprojekten wie KLIWAS (Bülow et al. 2014), KLIFF (Moseley et al. 2012), KLIMZUG-NORD (Rechid et al. 2014b, 2014c) und RADOST (Martinez und Blobel 2014) zur Verfügung. Zudem sind seit 2013 weitere Fachartikel erschienen, die das Klima und den Klimawandel in der Region thematisieren. Bezüglich der Veröffentlichungen ist insbesondere der 2015 veröffentlichte Klimabericht für den Ostseeraum (BACC II Author Team 2015) zu nennen, der ähnlich wie der Hamburger Klimabericht (HKB) den Stand des Wissens zum regionalen Klima sowie dessen zeitliche Veränderungen und Folgen für den Ostseeraum dokumentiert. Zudem wurden neben neuen Fachartikeln webbasierte Informationsangebote entwickelt, die das Klima und den Klimawandel in der Region thematisieren (• Tab. 2.1).

\subsection{Klimazustand}

Durch lange homogene In-situ-Messreihen lässt sich der Klimazustand eines bestimmten Ortes am besten bestimmen. Neben der räumlichen Variabilität der Messgrößen entscheiden vor allem der Standort der Messstation sowie die Beschaffenheit und Änderungen des direkten Messumfeldes über die Repräsentativität der Messungen für eine größere Umgebung. Messnetze unterschiedlicher Stationsdichte tragen dieser Tatsache Rechnung. Datensätze aus räumlich interpolierten Messdaten geben Informationen für die Fläche. Neben den In-situ-Beobachtungen haben sich in den letzten Jahrzehnten zunehmend Fernerkundungsverfahren und Reanalysen etabliert (American Meteorological Society 2012). Letztere beruhen auf numerischen Atmosphärenmodellen und bieten Gitterpunktfelder von physikalisch konsistenten meteorologischen Größen mit einer größeren Vielfalt an Parametern auch in verschiedenen Höhen der Atmosphäre und in hoher zeitlicher Auflösung. Jedes Verfahren hat seine Stärken und Schwächen, und so können die verschiedenen methodischen Ansätze zu Unterschieden in den Datensätzen führen. Die voneinander abweichenden Werte zeigen die Unschärfe beispielsweise bei der Bestimmung des Klimazustandes auf. Sowohl die Messreihen als auch die Zeitreihen der Reanalysen können Inhomogenitäten aufweisen, die bei den Messreihen auf Stationsverlegungen, Änderungen von Messgeräten und Beobachtungsvorschriften oder auf Veränderungen in der Stationsumgebung zurückzuführen sind. In den Reanalysen können Inhomogenitäten durch eine sich zeitlich verändernde Datenbasis erfolgen, indem neue Datenquellen wie Satelliten- oder Radardaten assimiliert werden. Auch wenn es sich bei den Reanalysen um homogenisierte Datensätze handelt, ist weiterhin zu berücksichtigen, dass es sich um Modelldaten handelt, die kein exaktes Abbild der Realität liefern können. Zudem ist die Unterschiedlichkeit der räumlichen Repräsentativität der verschiedenen Datensätze zu beachten. Während es sich bei den Beobachtungsdaten um Punktmessungen handelt, denen eine gewisse Repräsentativität für das Umfeld zugesprochen wird, beziehen sich die Werte der räumlich homogenen Reanalysen auf Modellgitterpunkte, deren räumliche Auflösung je nach Modelllauf variiert.

- Tab. 2.1 Webbasierte Informationsangebote zum Klimawandel in der MRH und Norddeutschland

\begin{tabular}{|c|c|c|}
\hline Name & Anbieter & Inhalt \\
\hline Climate Data Center & DWD & $\begin{array}{l}\text { 30-jährige Mittelwerte von DWD-Stationsdaten, } \\
\text { phänologischen Daten, abgeleiteten Parametern, } \\
\text { Rasterfelder }\end{array}$ \\
\hline Deutscher Klimaatlas & DWD & $\begin{array}{l}\text { Rasterkarten und Zeitreihen meteorologischer } \\
\text { Größen } \\
\text { Vergangenheit und Zukunft }\end{array}$ \\
\hline $\begin{array}{l}\text { Dokumentenserver } \\
\text { Klimawandel }\end{array}$ & GERICS, SUB Hamburg & Dokumentenarchiv der KLIMZUG-Projekte \\
\hline Klimanavigator & $\begin{array}{l}\text { Nationales Portal, GERICS \& } \\
\text { Partner }\end{array}$ & $\begin{array}{l}\text { Überblick zu klimarelevanter Forschung und } \\
\text { Klimawandel }\end{array}$ \\
\hline $\begin{array}{l}\text { Norddeutscher } \\
\text { Klimaatlas }\end{array}$ & $\begin{array}{l}\text { Norddeutsches Küsten- und } \\
\text { Klimabüro/Institut für Küsten- } \\
\text { forschung/HZG }\end{array}$ & $\begin{array}{l}\text { Analysen und Interpretationen von regionalen } \\
\text { Klimaprojektionen bis } 2100 \text { in Norddeutschland }\end{array}$ \\
\hline $\begin{array}{l}\text { Norddeutscher } \\
\text { Klimamonitor }\end{array}$ & $\begin{array}{l}\text { Norddeutsches Küsten- und } \\
\text { Klimabüro/Institut für Küsten- } \\
\text { forschung/HZG und DWD }\end{array}$ & $\begin{array}{l}\text { Analysen und Interpretationen von Stationsdaten, } \\
\text { Rasterkarten und Reanalysen bzgl. Klimazustand, } \\
\text { bisherigen Klimaentwicklungen und Konsistenz mit } \\
\text { regionalen Klimaprojektionen in Norddeutschland }\end{array}$ \\
\hline Regionaler Klimaatlas & $\begin{array}{l}\text { Regionale Klimabüros der } \\
\text { Helmholtz-Gemeinschaft }\end{array}$ & $\begin{array}{l}\text { Analysen und Interpretationen von regionalen } \\
\text { Klimaprojektionen bis } 2100 \text { für alle Bundesländer }\end{array}$ \\
\hline
\end{tabular}

\section{Literatur/Website}

Kaspar et al. 2013 www.dwd.de/cdc

Kaspar et al. 2013 www.deutscher-klimaatlas.de www.edoc.sub.uni-hamburg.de/ klimawandel/home

www.klimanavigator.de

Meinke und Gerstner 2009 www.norddeutscherklimaatlas.de

Meinke et al. 2014 www.norddeutscherklimamonitor.de

Meinke et al. 2010 www.regionaler-klimaatlas.de 
- Abb. 2.1 Jahresmittel der Windgeschwindigkeit in $\mathrm{m} / \mathrm{s}$ im Bereich der Metropolregion Hamburg 1981-2010. Dargestellt ist die räumliche Verteilung gemäß der Reanalyse coastDat2 (Geyer 2014). Die Punkte markieren die Lage von Klimastationen des DWD (rot $=$ Stationen mit hinreichend wenigen Fehlwerten im Zeitraum 1981-2010, grau = Stationen mit zu vielen Fehlwerten. (Norddeutscher Klimamonitor, Meinke et al. 2014)

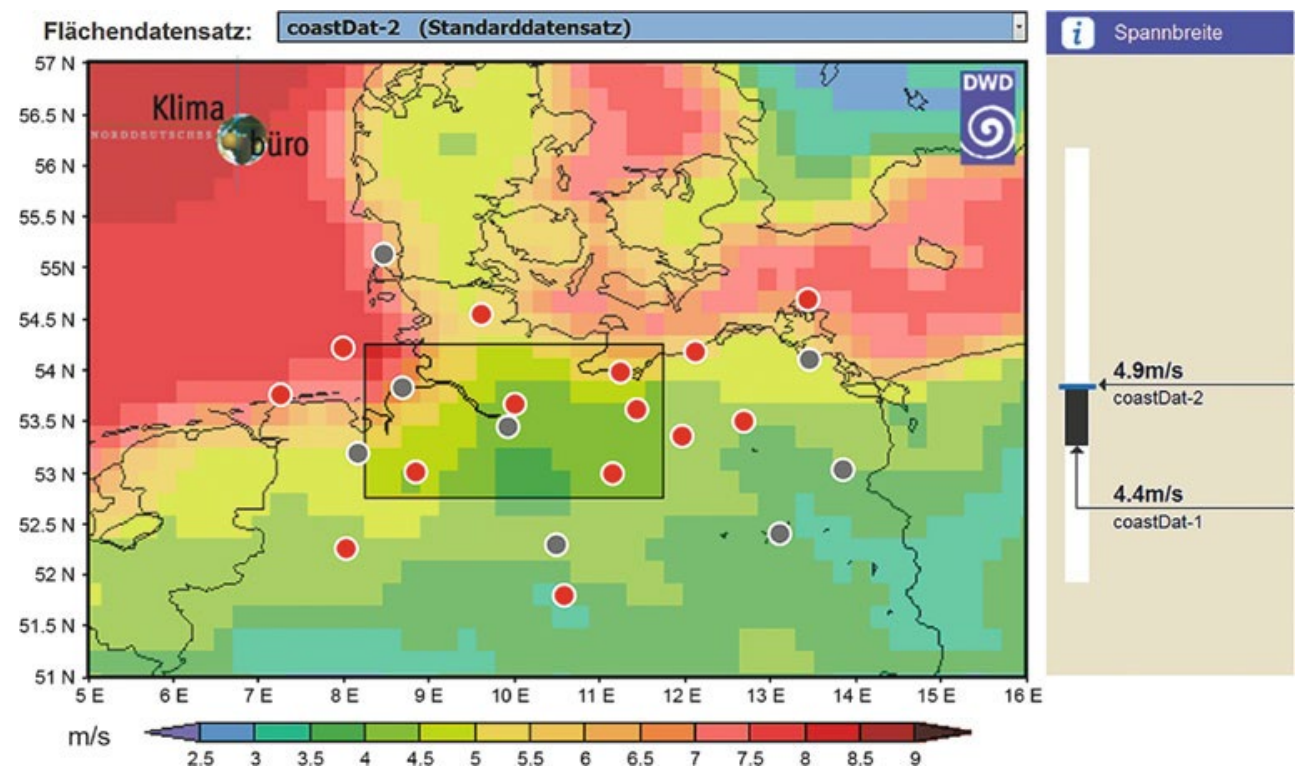

Den nachfolgenden Ausführungen liegt die Klimabeschreibung der Metropolregion Hamburg (Rosenhagen und Schatzmann 2011) aus dem 1. HKB zugrunde. Die Auswertungen wurden durch Beobachtungsdaten des DWD (Kaspar et al. 2013), weiteren räumlich interpolierten Beobachtungsdaten und regionalen Reanalysen für den Zeitraum 1981-2010 aktualisiert. Die Ergebnisse werden mit den Auswertungen im Norddeutschen Klimamonitor (Stand Oktober 2015) verglichen. Dieser entstand in einer Kooperation des Norddeutschen Küsten- und Klimabüros am Helmholtz-Zentrum Geesthacht mit dem Deutschen Wetterdienst und basiert auf den Reanalysen coastDat1 und coastDat2, sechs Flächendatensätzen (CRU TS3.2, Matsuura-Willmott V3.01, EOBS 9.0, DWD NKDZ, DWD REGNIE, GPCC v6) sowie 22 Messstationen des DWD-Messnetzes (Stand Oktober 2015 ${ }^{1}$, Meinke et al. 2014).

\subsubsection{Wind}

Der Wind ist eines der die Metropolregion Hamburg am stärksten prägenden meteorologischen Elemente. Einst hatte die Seefahrt unter Segeln die Küsten- und handeltreibenden Städte reich gemacht, den Menschen Fortschritt und eine Verbindung mit der ganzen Welt gebracht; heute ist Norddeutschland mit tausenden Windkraftwerken auf dem Weg zum Zentrum einer neuen regenerativen Energiewirtschaft.

Das Jahresmittel der Windgeschwindigkeit in $10 \mathrm{~m}$ Höhe über Grund (1981-2010) liegt - je nach Datengrundlage - in der Hamburger Metropolregion (Gebietsmittel über Land- und Meeresflächen) bei 4,5-5 m/s. Räumlich nimmt die jährliche mittlere Windgeschwindigkeit von 3-5 m/s im Landesinnern auf 5-7 m/s an den Küsten zu. Bezogen auf die Stationsmessungen in der Hamburger Metropolregion ist sie mit $8 \mathrm{~m} / \mathrm{s}$ auf Helgoland in der Deutschen Bucht am höchsten und mit $3 \mathrm{~m} / \mathrm{s}$ in Lüchow am

1 Der Norddeutsche Klimamonitor wurde im Frühjahr 2017 aktualisiert. Dabei wurde der Datenbestand um den Zeitraum 2011 bis 2015 erweitert. (Meinke 2017). niedrigsten (Norddeutscher Klimamonitor, Meinke et al. 2014); vgl. auch - Abb. 2.1, in der die räumliche Struktur auf Basis der Reanalyse coastDat2 (Geyer 2014) gezeigt wird. Im jahreszeitlichen Verlauf weist die mittlere Windgeschwindigkeit (1981-2010) in der Metropolregion Hamburg mit Werten von rund 4-4,5 m/s (je nach Datensatz) ein Minimum im Sommer (Juni, Juli, August) auf. Im Winter (Dezember, Januar, Februar) zeigen die mittleren Windgeschwindigkeiten maximale Werte etwa zwischen 5 und $5,5 \mathrm{~m} / \mathrm{s}$ (Norddeutscher Klimamonitor, Meinke et al. 2014).

Hohe Windgeschwindigkeiten werden vor allem im Winterhalbjahr von den außertropischen Tiefdruckgebieten ausgelöst, die vom Nordatlantik auf Europa übergreifen. In den letzten Jahren wurden sehr hohe Windgeschwindigkeiten im Bereich der dicht aufeinanderfolgenden Orkane CHRISTIAN (28.10.2013) und XAVER (5.-7.12.2013) registriert (von Storch et al. 2014; Leiding et al. 2014). Bei CHRISTIAN trat an der Station Sankt-Peter-Ording mit einer Spitzenbö von $172 \mathrm{~km} / \mathrm{h}$ die höchste Windgeschwindigkeit dieses Ereignisses in Deutschland auf. Bemerkenswert ist ebenfalls, dass im nördlichen Schleswig-Holstein auch im 10-minMittel volle Orkanstärke herrschte (Büsum $127 \mathrm{~km} / \mathrm{h}$, Haeseler und Lefebvre 2013). Beim Orkan XAVER kam es zu einer Kettensturmflut an der deutschen Nordseeküste mit Wasserständen von 2,5 bis knapp $4 \mathrm{~m}$ über dem mittleren Hochwasser (BSH 2013; Deutschländer et al. 2013, vgl. \ Kap. 4). Hohe Windgeschwindigkeiten treten auch im Bereich sommerlicher Gewitter auf. Diese haben ebenfalls ein großes Schadenspotenzial, da sie oft mit Starkregen oder Hagel verbunden sind, hin und wieder aber auch zur Ausbildung von Tornados führen, wie am 05.05.2015 knapp östlich der Metropolregion (Haeseler et al. 2015).

Einen Überblick über die Häufigkeit der höchsten täglichen Windgeschwindigkeiten im Verlauf eines Jahres gibt 0 Tab. 2.2. Sie zeigt, dass an Standorten mit hohen jährlichen Windgeschwindigkeiten wie auf See auch häufiger hohe Spitzenböen auftreten als an windärmeren Standorten im Binnenland. Schwere Sturm- und Orkanböen mit Windgeschwindigkeiten von mehr als $24,5 \mathrm{~m} / \mathrm{s}$ kommen auf Helgoland im Mittel an 26 Tagen im Jahr vor, an der Elbmündung (Cuxhaven) an knapp 12 Tagen, an 
- Tab. 2.2 Mittlere Häufigkeit (Tage pro Jahr) der täglichen Spitzenböen im Zeitraum 1981-2010. (Quelle: eigene Auswertung auf der Grundlage der Daten des Climate Data Center des DWD, teilweise ergänzt mit Daten der DWD-Datenbank MIRAKEL)

\begin{tabular}{|c|c|c|c|c|c|c|c|}
\hline $\mathrm{Bft}$ & $\begin{array}{l}\text { Windgeschwindigkeit } \\
\text { in } \mathrm{m} / \mathrm{s}\end{array}$ & Helgoland & Cuxhaven & $\begin{array}{l}\text { Hamburg- } \\
\text { Fuhlsbüttel }\end{array}$ & Boltenhagen & Schwerin & Lüchow \\
\hline 0 & $0-0,2$ & 0,0 & 0,0 & 0,0 & 0,0 & 0,0 & 0,0 \\
\hline 1 & $0,3-1,5$ & 0,0 & 0,0 & 0,1 & 0,0 & 0,0 & 0,2 \\
\hline 2 & $1,6-3,3$ & 0,0 & 0,1 & 1,3 & 0,0 & 0,6 & 5,3 \\
\hline 3 & $3,4-5,4$ & 1,5 & 3,8 & 16,5 & 7,6 & 11,7 & 39,2 \\
\hline 4 & $5,5-7,9$ & 12,3 & 33,9 & 61,4 & 37,6 & 69,5 & 95,6 \\
\hline 5 & $8,0-10,7$ & 57,9 & 83,5 & 106,3 & 89,2 & 104,9 & 108,3 \\
\hline 6 & $10,8-13,8$ & 73,6 & 95,8 & 97,4 & 98,4 & 88,9 & 66,4 \\
\hline 7 & $13,9-17,1$ & 92,0 & 75,1 & 53,0 & 71,1 & 50,4 & 32,2 \\
\hline 8 & $17,2-20,7$ & 65,5 & 41,5 & 20,6 & 34,7 & 20,5 & 12,0 \\
\hline 9 & $20,8-24,4$ & 35,6 & 18,0 & 5,5 & 14,2 & 8,5 & 3,5 \\
\hline 10 & $24,5-28,4$ & 18,7 & 8,2 & 2,2 & 4,5 & 3,5 & 1,4 \\
\hline 11 & $28,5-32,6$ & 5,2 & 2,3 & 0,4 & 1,7 & 1,0 & 0,4 \\
\hline 12 & 32,7 und mehr & 2,0 & 1,1 & 0,2 & 0,9 & 0,6 & 0,1 \\
\hline
\end{tabular}

- Tab. 2.3 Monats- und Jahresmittel der Lufttemperatur in ${ }^{\circ} \mathrm{C}$ von Stationen in der Metropolregion Hamburg für die Referenzperiode $1981-2010$. (Quelle: Climate Data Center des DWD)

\begin{tabular}{|c|c|c|c|c|c|c|c|c|c|c|c|c|c|}
\hline Station & Jan & Feb & Mrz & Apr & Mai & Jun & Jul & Aug & Sep & Okt & Nov & Dez & Jahr \\
\hline Boltenhagen & 1,4 & 1,6 & 4,0 & 7,4 & 11,7 & 14,8 & 17,5 & 17,5 & 14,2 & 10,0 & 5,4 & 2,2 & 9,0 \\
\hline Cuxhaven & 2,2 & 2,3 & 4,7 & 8,3 & 12,4 & 15,2 & 17,7 & 17,7 & 14,7 & 10,6 & 6,2 & 2,9 & 9,6 \\
\hline $\begin{array}{l}\text { Hamburg- } \\
\text { Fuhlsbüttel }\end{array}$ & 1,6 & 1,9 & 4,6 & 8,6 & 12,9 & 15,6 & 18,1 & 17,6 & 14,0 & 9,8 & 5,4 & 2,2 & 9,4 \\
\hline Helgoland & 3,4 & 2,8 & 4,3 & 7,1 & 10,9 & 14,0 & 16,8 & 17,4 & 15,3 & 11,9 & 7,8 & 4,7 & 9,7 \\
\hline Lüchow & 0,9 & 1,3 & 4,5 & 8,6 & 13,3 & 15,8 & 18,3 & 17,7 & 13,8 & 9,3 & 4,9 & 1,6 & 9,2 \\
\hline Schwerin & 0,7 & 1,2 & 4,1 & 8,3 & 12,9 & 15,5 & 18,1 & 17,7 & 13,9 & 9,5 & 4,8 & 1,5 & 9,0 \\
\hline
\end{tabular}

der Ostseeküste bei Boltenhagen an 9 Tagen, weiter landeinwärts an 5 Tagen pro Jahr oder seltener. Dies stimmt mit den Ergebnissen des Norddeutschen Klimamonitors überein, wonach die jährliche Anzahl der Sturmtage (maximale Windgeschwindigkeit in Böen überschreitet $17,2 \mathrm{~m} / \mathrm{s}$; entsprechend Beaufort-Skala $8=$ stürmischer Wind) über See mit beispielsweise 126 Sturmtagen pro Jahr auf Helgoland am größten ist, während die Sturmhäufigkeiten im Landesinnern deutlich geringer ausfallen, so z. B. in Lüchow, wo im Mittel nur 17 Sturmtage pro Jahr auftreten. Im Jahresverlauf treten die meisten Sturmtage im Winter auf, im Sommer ist die Sturmhäufigkeit in der Hamburger Metropolregion am geringsten. Die Häufigkeit windstiller Tage ist komplementär auf See gering und im Landesinnern etwas häufiger, jedoch ohne klaren Jahresgang (Meinke et al. 2014).

\subsubsection{Lufttemperatur}

In der Metropolregion Hamburg weisen die Messstationen im Bereich der Deutschen Bucht die höchsten Jahresmittelwerte der 2-m-Temperaturen auf (• Tab. 2.3). In Richtung Südosten nehmen sie leicht ab. Der Jahresgang der Temperatur ist durch die Abnahme des maritimen Einflusses mit der Entfernung von Nord- und Ostsee und nach Osten hin durch zunehmende Kontinentalität geprägt. Im Vergleich zu den Küsten und Inseln werden die Sommer nach Süden hin wärmer und die Winter kälter.

Die räumliche Struktur der jährlichen Temperaturverteilung für den Zeitraum 1981-2010 wird im Norddeutschen Klimamonitor (Meinke et al. 2014) anhand der Analysen von derzeit (Stand Oktober 2015) vier interpolierten Klimadatensätzen und zwei Reanalysen angezeigt, von denen $\mathbf{0}$ Abb. 2.2 den sogenannten Standarddatensatz zeigt. Mit dem Ziel einer realistischen Darstellung wurde im Norddeutschen Klimamonitor je Aspekt (Klimazustand, Klimaentwicklung und Konsistenztest) für jede meteorologische Größe ein Standarddatensatz identifiziert. Das Gebietsmittel dieses Standarddatensatzes weist über alle Jahreszeiten die kleinste Differenz sowohl a) zum Ensemble-Mittel der im Klimamonitor zugrunde liegenden Flächendaten für Norddeutschland auf als auch b) zum Mittel der vollständig vorliegenden Stationen in Norddeutschland. Für alle meteorologischen 
- Abb. 2.2 Jahresmittel der Lufttemperatur in ${ }^{\circ} \mathrm{C}$ gemäß der Klimatologie Matsuura-Willmott V3 (Willmott und Matsuura 2012). Die Punkte markieren die Lage von Klimastationen des DWD (rot = Stationen mit hinreichend wenigen Fehlwerten im Zeitraum 1981-2010, grau $=$ Stationen mit zu vielen Fehlwerten. (Norddeutscher Klimamonitor, Meinke et al. 2014)

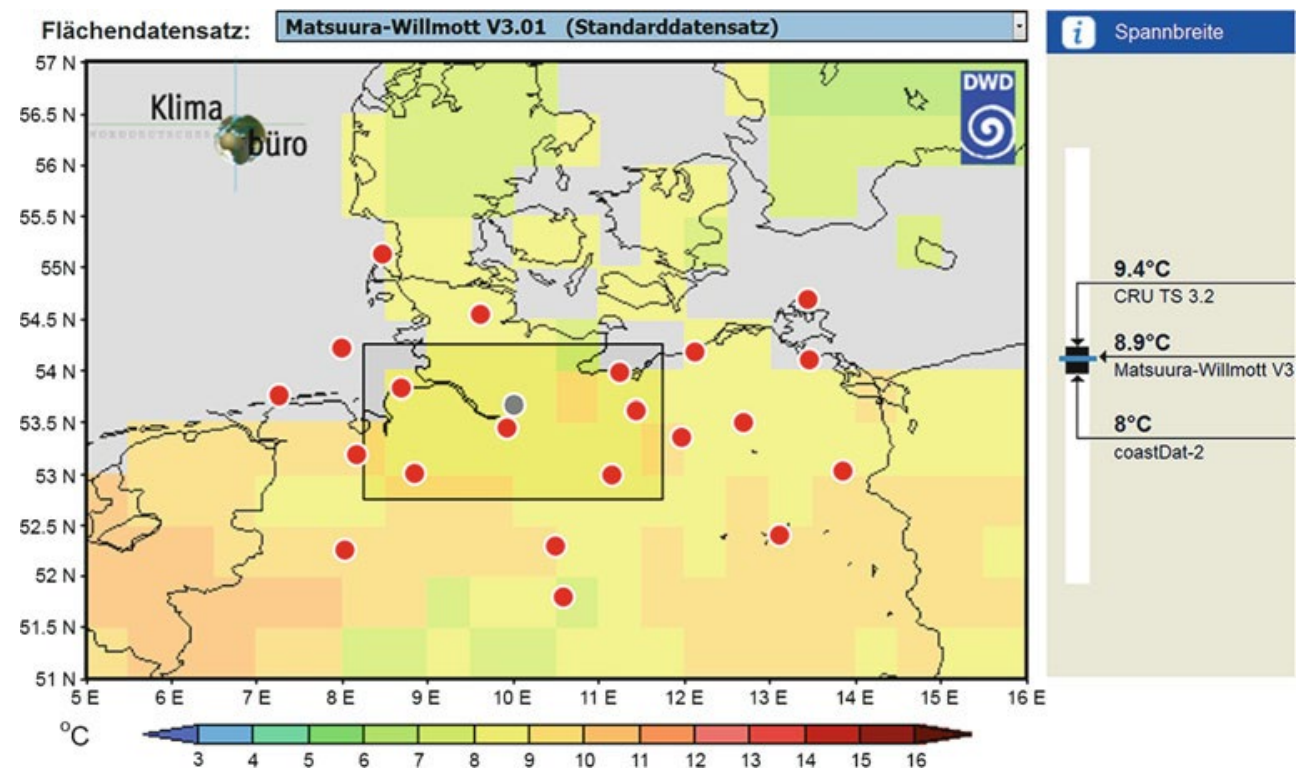

- Tab. 2.4 Mittlere monatliche und jährliche Anzahl von Ereignistagen an der Station Hamburg-Fuhlsbüttel für die Referenzperiode $1981-2010$. (Quelle: Climate Data Center des DWD)

\begin{tabular}{|c|c|c|c|c|c|c|c|c|c|c|c|c|c|}
\hline Station & Jan & Feb & Mrz & Apr & Mai & Jun & Jul & Aug & Sep & Okt & Nov & Dez & Jahr \\
\hline Eistag & 6,1 & 3,7 & 0,6 & 0,0 & 0,0 & 0,0 & 0,0 & 0,0 & 0,0 & 0,0 & 0,7 & 5,2 & 16,4 \\
\hline Frosttag & 15,1 & 14,5 & 10,6 & 4,4 & 0,3 & 0,0 & 0,0 & 0,0 & 0,0 & 2,4 & 7,7 & 15,0 & 70,0 \\
\hline Sommertag & 0,0 & 0,0 & 0,0 & 0,5 & 2,3 & 4,6 & 9,5 & 8,2 & 1,5 & 0,0 & 0,0 & 0,0 & 26,5 \\
\hline Heißer Tag & 0,0 & 0,0 & 0,0 & 0,0 & 0,0 & 0,6 & 2,3 & 1,4 & 0,0 & 0,0 & 0,0 & 0,0 & 4,5 \\
\hline Tropennacht & 0,0 & 0,0 & 0,0 & 0,0 & 0,0 & 0,0 & 0,5 & 0,3 & 0,0 & 0,0 & 0,0 & 0,0 & 0,8 \\
\hline
\end{tabular}

Größen, für die keine Beobachtungsstationen über den gesamten Untersuchungszeitraum vorhanden sind, wurde im Norddeutschen Klimamonitor coastDat-2 als Standarddatensatz festgelegt, da dieser Datensatz die längste homogene Zeitreihe aufweist.

Entsprechend dem Standarddatensatz liegt das Gebietsmittel der Temperatur für die Metropolregion Hamburg im Zeitraum 1981-2010 bei etwa $9^{\circ} \mathrm{C}$. Die Spannbreite aus den Gebietsmittelwerten aller verwendeten Datensätze reicht in dieser Region von 8 bis $9,4^{\circ} \mathrm{C}$ (vgl. - Abb. 2.2). Mittlere Lufttemperaturen liegen im Sommer bei etwa $17^{\circ} \mathrm{C}$, während sie im Winter Mittelwerte von etwa $1,5^{\circ} \mathrm{C}$ aufweisen. Im Frühjahr und Herbst liegen die mittleren Lufttemperaturen nahe den Jahresmittelwerten (Meinke et al. 2014).

Trusilova und Riecke (2015) analysieren Gebietsmittel der Metropolregion Hamburg aus einem räumlich interpolierten Flächendatensatz in verschiedenen 30-jährigen Bezugszeiträumen. Der jüngste betrachtete Zeitraum umfasst die Jahre 1981-2010. Das Flächenmittel der Jahrestemperatur des Bezugszeitraums 1981-2010 beträgt hier $9,4^{\circ} \mathrm{C}$ und liegt im Vergleich zu 19712000 um $0,2{ }^{\circ} \mathrm{C}$ und in Relation zu $1961-1990$ um $0,6^{\circ} \mathrm{C}$ höher. Ähnliche Ergebnisse zeigen die Auswertungen des Norddeutschen Klimamonitors (vgl. Meinke et al. 2014).

Die räumliche Verteilung von Tagen mit Lufttemperaturen ober- und unterhalb markanter Schwellenwerte (Ereignistage) hängt eng mit dem Monatsmittel der Lufttemperatur zusammen (s. DWD-Klimaatlas, Norddeutscher Klimamonitor). Durch den auf den Jahres- und Tagesgang der Temperatur dämpfenden Einfluss von Nord- und Ostsee ist die Zahl der Sommer- und heißen Tage und der Frosttage auf Helgoland und direkt an den Küsten am geringsten und in der Lüneburger Heide am größten. Hamburg (vgl. - Tab. 2.4) liegt mit etwa 27 Sommertagen (Tagestemperaturmaximum $\geq 25^{\circ} \mathrm{C}$ ) pro Jahr in der Mitte der räumlichen Spannweite, die sich zwischen zwei Sommertagen auf Helgoland, 11-13 an den Küsten bei Cuxhaven bzw. Boltenhagen und 36 Sommertagen in der Lüneburger Heide (Lüchow) erstreckt (Norddeutscher Klimamonitor, Meinke et al. 2014). Heiße Tage (max. Tagestemperatur $\geq 30^{\circ} \mathrm{C}$ ) treten derzeit in der Hamburger Metropolregion im Mittel etwa fünfmal pro Jahr auf, wobei es auch hier deutliche Unterschiede zwischen Küste (z. B. Cuxhaven mit zwei heißen Tagen) und Binnenland (z. B. Lüchow mit acht heißen Tagen) gibt. Tropische Nächte (tiefste Nachttemperatur $\geq 20^{\circ} \mathrm{C}$ ) treten derzeit ausschließlich im Sommer (Juni bis August) auf. Während die Häufigkeit heißer Tage landeinwärts zunimmt, sind tropische Nächte an der Küste häufiger. So gibt es derzeit in Cuxhaven etwa eine tropische Nacht pro Jahr, während beispielsweise in Lüchow seit Messbeginn im November 1971 keine einzige tropische Nacht verzeichnet wurde. In HamburgNeuwiedenthal kamen sie im Zeitraum 1981-2010 im Mittel etwa alle zwei Jahre einmal vor (Meinke et al. 2014). Trusilova und Riecke (2015) finden im Zeitraum 1989-2008 in der Stadt Ham- 
- Tab. 2.5 Mittlere Monats- und Jahressummen der Niederschlagshöhen in mm von Stationen in der Metropolregion Hamburg für die Referenzperiode 1981-2010. (Quelle: Climate Data Center des DWD)

\begin{tabular}{|c|c|c|c|c|c|c|c|c|c|c|c|c|c|}
\hline Station & Jan & Feb & Mrz & Apr & Mai & Jun & Jul & Aug & Sep & Okt & Nov & Dez & Jahr \\
\hline Boltenhagen & 43,8 & 34,0 & 41,2 & 33,8 & 53,0 & 63,4 & 61,1 & 72,1 & 50,0 & 45,7 & 45,5 & 48,1 & 591,7 \\
\hline Cuxhaven & 67,3 & 47,1 & 56,4 & 38,4 & 51,6 & 79,7 & 80,9 & 81,3 & 86,5 & 90,0 & 79,7 & 71,9 & 830,8 \\
\hline $\begin{array}{l}\text { Hamburg- } \\
\text { Fuhlsbüttel }\end{array}$ & 67,8 & 49,9 & 68,5 & 43,0 & 57,4 & 78,6 & 76,7 & 78,9 & 67,4 & 67,0 & 69,2 & 68,9 & 793,3 \\
\hline Helgoland & 58,4 & 42,5 & 49,5 & 33,9 & 41,6 & 54,4 & 63,6 & 79,7 & 87,4 & 87,0 & 78,9 & 67,5 & 744,4 \\
\hline Lüchow & 46,0 & 34,5 & 41,2 & 32,3 & 50,0 & 53,0 & 66,6 & 59,1 & 45,5 & 41,6 & 43,0 & 43,9 & 556,7 \\
\hline Schwerin & 53,6 & 40,5 & 48,5 & 38,8 & 52,4 & 61,1 & 69,7 & 62,9 & 55,3 & 50,6 & 51,3 & 55,4 & 640,1 \\
\hline
\end{tabular}

burg bis zu fünf Tropennächte in einem Jahr. Im Bezugszeitraum 1981-2010 traten in der Metropolregion drei Hitzeperioden auf, wenn diese Ereignisse an Hamburg festgemacht werden. Dabei trat die erste und zugleich stärkste seit $1950 \mathrm{im}$ Sommer 1994 auf (Imbery et al. 2015). Imbery et al. (2015) definieren die Hitzeperiode als einen 14-tägigen Zeitraum mit einem mittleren Tagesmaximum der Lufttemperatur von mindestens $30^{\circ} \mathrm{C}$. Frost- und Eistage treten in der Hamburger Metropolregion derzeit (19812010) ganzjährig, außer im Sommer (Juni bis August) auf. Ein typischer Winter besteht derzeit etwa zur Hälfte aus Frosttagen (Temperaturminimum $<0^{\circ} \mathrm{C}$ ), wobei davon an etwa 14 Tagen Eistage auftreten (max. Tagestemperatur $<0{ }^{\circ} \mathrm{C}$ ).

\subsubsection{Niederschlag}

Wie bereits bei Rosenhagen und Schatzmann (2011) ausgeführt, nimmt die jährliche Niederschlagshöhe mit zunehmender Kontinentalität im Mittel von West nach Ost ab, was sich sowohl in den Messdaten in $\mathbf{0}$ Tab. 2.5 als auch in den interpolierten Messdatenanalysen und Reanalysen widerspiegelt, die im Norddeutschen Klimamonitor dargestellt sind. Entsprechend dem Standarddatensatz des Norddeutschen Klimamonitors (Beschreibung s. o.) liegt die mittlere Niederschlagssumme (1981-2010) in der Metropolregion Hamburg bei etwa 720 mm (Gebietsmittel über Landflächen). Vergleicht man die gemessenen jährlichen Niederschlagshöhen an den Messstationen in der Metropolregion, wird deutlich, dass an der Küste, z. B. an der Station Cuxhaven, mit $831 \mathrm{~mm}$ deutlich mehr Niederschlag fällt als im Binnenland, wie z. B. in der Lüneburger Heide an der Station Lüchow $(557 \mathrm{~mm})$. Im Vergleich zum Zeitraum 1971-2000 (Rosenhagen und Schatzmann 2011) haben die mittleren Jahresniederschläge um rund $5 \%$ zugenommen. Dabei zeigen sich im jahreszeitlichen Wechsel kaum Unterschiede zwischen den beiden Zeiträumen (1971-2000 und 1981-2010). Das Niederschlagsmaximum liegt in der Metropolregion Hamburg im Sommer bei etwa $220 \mathrm{~mm}$. Am wenigsten Niederschlag fällt hier im Frühjahr mit etwa $150 \mathrm{~mm}$ (Norddeutscher Klimamonitor; Meinke et al. 2014). Bezogen auf einzelne Monate zeigt der Jahresgang jedoch markante Unterschiede. Im Zeitraum 1981-2010 ist der trockenste Monat an allen Stationen der April, während es im Zeitraum 1971-2000 meist noch der Februar war (Rosenhagen und Schatzmann 2011).
$\mathrm{Zu}$ dieser Veränderung trug insbesondere der April 2007 bei - der trockenste April seit 1893, in dem in der Metropolregion durchweg weniger als $10 \mathrm{~mm}$, regional sogar nur $1 \mathrm{~mm}$ Niederschlag fiel (Müller-Westermeier et al. 2008). Aber auch in den Jahren 2009-2011 war der April deutlich zu trocken. Die Niederschlagsmaxima sind an den Stationen auf verschiedene Jahreszeiten verteilt. An den Stationen Helgoland und Cuxhaven fallen sie auf die Herbstmonate (Helgoland im September, Cuxhaven im Oktober), an den anderen Stationen auf die Sommermonate Juli und August. Auffällig ist die zwischen beiden Bezugszeiträumen teilweise kräftige Zunahme der Niederschläge im August um meist 10-20 mm. Hohe tägliche Niederschlagshöhen können in der Hamburger Metropolregion in allen Jahreszeiten vorkommen. Im Sommerhalbjahr sind sie meist mit Gewittern verbunden. Innerhalb kurzer Zeit kann mehr als $100 \mathrm{~mm}$ Niederschlag fallen und für lokale Überschwemmungen sorgen. Im Winterhalbjahr sind es oft über mehrere Tage andauernde Niederschlagsereignisse, in Kombination mit gesättigten Böden oder einer schmelzenden Schneedecke, die für Hochwasser in den Flüssen sorgen. Besonders kritisch wirkt sich dies an den Nebengewässern der Tideelbe (z. B. Krückau und Este) aus, wenn gleichzeitig eine Sturmflut die Entwässerung der Nebenflüsse verhindert.

\section{- Schnee}

Die mittlere jährliche Anzahl der Tage mit Schneedecke liegt in der Metropolregion Hamburg zwischen 12 Tagen auf Helgoland und 25 Tagen im Bereich der Lüneburger Heide (• Tab. 2.6, Station Amelinghausen). Sie nimmt mit zunehmender Entfernung von der Nordseeküste zu und zeigt damit den mildernden Einfluss des Meeres in den Wintermonaten. Im Vergleich zum Zeitraum 1971-2000 (Rosenhagen und Schatzmann 2011) nahm die Anzahl der Tage mit Schneedecke in Hamburg um 5 zu. Dazu trug insbesondere das schneereiche Jahr 2010 bei, das mit $97 \mathrm{Ta}-$ gen (davon 31 im Januar und 26 im Februar) den Höchstwert des Zeitraums 1971-2000 mit 72 Tagen (1979) deutlich übertraf.

Insbesondere im Bereich der Ostseeküste kann es in Kombination von starken Schneefällen und hohen Windgeschwindigkeiten zu folgenreichen Schneeverwehungen kommen. Das stärkste Ereignis der letzten Jahrzehnte war der blizzardartige Wintereinbruch zum Jahreswechsel 1978/79, der mit meterhohen Schneeverwehungen und fast flächenhaft blockiertem Verkehr verbunden war. Eine teilweise vergleichbare Entwicklung gab es 
- Tab. 2.6 Mittlere monatliche und jährliche Anzahl von Tagen mit Schneedecke an Stationen in der Metropolregion Hamburg für die Referenzperiode 1981-2010. (Eigene Auswertung auf der Basis der Daten des Climate Data Center des DWD)

\begin{tabular}{|c|c|c|c|c|c|c|c|c|c|c|c|c|c|}
\hline Station & Jan & Feb & Mrz & Apr & Mai & Jun & Jul & Aug & Sep & Okt & Nov & Dez & Jahr \\
\hline Boltenhagen & 7,8 & 7,4 & 3,8 & 0,1 & 0 & 0 & 0 & 0 & 0 & 0,0 & 1,0 & 4,4 & 24,6 \\
\hline Cuxhaven & 7,0 & 5,9 & 2,9 & 0,2 & 0 & 0 & 0 & 0 & 0 & 0,0 & 0,9 & 4,7 & 21,6 \\
\hline $\begin{array}{l}\text { Hamburg- } \\
\text { Fuhlsbüttel }\end{array}$ & 8,9 & 7,4 & 3,6 & 0,3 & 0 & 0 & 0 & 0 & 0 & 0,0 & 1,6 & 6,2 & 28,0 \\
\hline Helgoland & 4,5 & 3,2 & 1,9 & 0,1 & 0 & 0 & 0 & 0 & 0 & 0,0 & 0,3 & 2,6 & 12,5 \\
\hline Amelinghausen & 8,4 & 7,4 & 3,0 & 0,1 & 0 & 0 & 0 & 0 & 0 & 0,1 & 1,5 & 5,1 & 25,6 \\
\hline Schwerin & 10,4 & 10,3 & 4,5 & 0,3 & 0 & 0 & 0 & 0 & 0 & 0,0 & 2,3 & 6,2 & 34,0 \\
\hline
\end{tabular}

- Tab. 2.7 Mittlere Monats- und Jahressummen der Sonnenscheindauer in h von Stationen in der Metropolregion Hamburg für die Referenzperiode 1981-2010. (Quelle: Climate Data Center des DWD)

\begin{tabular}{|l|l|l|l|l|l|l|l|l|l|}
\hline Station & Jan & Feb & Mrz & Apr & Mai & Jun & Jul & Aug & Sep \\
\hline Boltenhagen & 47,5 & 69,0 & 121,8 & 189,4 & 252,0 & 227,8 & 243,3 & 218,0 & 154,8 \\
\hline \\
\hline Cuxhaven
\end{tabular}

im Bereich der Sturmtiefs DAISY im Januar 2010. Auch hier kam es zu von der Außenwelt abgeschnittenen Dörfern, gesperrten Autobahnen sowie unterbrochenen Zug- und Fährverbindungen. Gleichzeitig trat an der Ostseeküste eine Sturmflut mit Schäden an Deichen und Infrastruktur auf (Booß et al. 2011).

\subsubsection{Sonnenscheindauer}

Die mittleren Jahressummen der Sonnenscheindauer der Referenzperiode 1981-2010 liegen an den DWD-Messstationen der Metropolregion zwischen 1580 und $1760 \mathrm{~h}$ (• Tab. 2.7) und sind mit Ausnahme von Hamburg - hier wurde eine leichte Abnahme verzeichnet - meist geringfügig $(<1 \%)$ höher als im Zeitraum 1971-2010 (Rosenhagen und Schatzmann 2011). Lediglich im Osten der Metropolregion schien die Sonne abseits der Küste im Mittel um knapp 10 \% mehr. Sonnenscheinbegünstigt sind Helgoland sowie die Küsten. Der sonnenscheinreichste Monat ist übereinstimmend der Mai, im Mittel scheint hier die Sonne an annähernd 7-8 h/Tag, während die Sonnenscheindauer im Dezember meist weniger als $2 \mathrm{~h} /$ Tag beträgt ( $\mathbf{0}$ Tab. 2.7). Im Vergleich zum Zeitraum 1971-2000 steht einer Zunahme um rund $10 \mathrm{~h}$ im April eine entsprechende Abnahme der Sonnenscheindauer im August gegenüber. Die Werte im Norddeutschen Klimamonitor, die auf den Reanalysen coastDat 1 und coastDat2 basieren, liegen mit Flächenmittelwerten von 2150 bzw. $1900 \mathrm{~h}$ deutlich über den Messwerten an den einzelnen Stationen. Dies könnte mit der unterschiedlichen Bestimmungsmethode der
Sonnenscheindauer zusammenhängen. Demgegenüber können die stationsbasierten Aussagen hinsichtlich der jahreszeitlichen Verteilung der Sonnenscheindauer durch die Gebietsmittelwerte für die Metropolregion Hamburg und Norddeutschland bestätigt werden. Wie auch die Messstationen zeigen, ist der Frühling in der gesamten Metropolregion im Mittel deutlich sonnenreicher als der Herbst (Meinke et al. 2014).

\subsection{Bisherige klimatische Entwicklung in der Region}

In diesem Abschnitt wird die bisherige klimatische Entwicklung in der Region beschrieben. Dabei basieren die Aussagen vor 1950 auf Veröffentlichungen, die vereinzelte, teils lückenhafte Stationsdaten auswerten. Auswertungen ab 1950 basieren auf einem dichteren Messnetz mit weniger Lücken, Reanalysen der Atmosphäre und Flächendaten aus interpolierten Beobachtungsdaten (vgl. - Abschn. 2.2). Der inhaltliche Fokus des Abschnitts liegt auf den bisherigen Schwankungen und Änderungen der großräumigen atmosphärischen Zirkulation sowie der oberflächennahen Klimaelemente wie Wind, Temperatur und Niederschlag und den daraus abgeleiteten Größen. Dabei werden im Zuge der Dokumentation wissenschaftlicher Erkenntnisse auch jeweils Bezüge zum Klimabericht im Ostseeraum (BACC II Author Team 2015) hergestellt, da ein Teil der Hamburger Metropolregion zum Ostseeeinzugsgebiet gehört und auf diese Weise ein übergeordneter regionaler Kontext hergestellt werden kann. Außerdem werden die Aussagen 
des 1. HKB aufgegriffen und neueren Arbeiten (Stand Oktober 2015) gegenübergestellt.

\subsubsection{Die atmosphärische Zirkulation}

Der Luftdruckunterschied zwischen den subpolaren und subtropischen Drucksystemen bestimmt die Nordatlantische Oszillation, die für das Klima in Nord- und Mitteleuropa sowie im Nordostatlantik eine wichtige Rolle spielt und damit auch das Klima in Norddeutschland beeinflusst. Die Intensität dieses Druckgefälles über dem Nordatlantik wird durch den Nordatlantischen Oszillationsindex (NAO-Index) ausgedrückt. Hohe Indexwerte sind mit einer intensiven nordatlantischen Westdrift verbunden, durch die im Winter milde Atlantikluft nach Europa advehiert wird. Ein hoher NAO-Index ist daher mit erhöhten Wintertemperaturen in Norddeutschland verbunden. Bei negativem NAO-Index ist die atlantische Westdrift schwach, sodass die winterliche Kaltluftproduktion über dem Kontinent weniger gestört wird. Dies führt zu unterdurchschnittlichen Wintertemperaturen in Norddeutschland (DWD 2015). Langfristig unterliegt die Nordatlantische Oszillation unregelmäßigen jährlichen und dekadischen Schwankungen. Innerhalb der letzten 50 Jahre wurden jedoch Phasen mit spezifischen Trends beobachtet: So wurde ab Mitte der 1960er-Jahre ein positiver Trend des NAOIndex beobachtet. Dies ging mit zonaler Zirkulation, milden und regenreichen Wintern sowie erhöhter Sturmaktivität einher (BACC II Author Team 2015; Hurrel et al. 2003). Ab Mitte der 1990er-Jahre traten dann vermehrt negative NAO-Indizes auf, mit denen eine verstärkt meridionale Zirkulation einhergeht (BACC II Author Team 2015).

Viele Studien untersuchen die Langzeitänderungen der atmosphärischen Zirkulation; sie stimmen in der Erkenntnis überein, dass sich die Zugbahnen von Tiefdruckgebieten über dem Nordatlantik nach Nordosten verlagert haben (BACC II Author Team 2015, S. 73). Diese Verlagerung ist konsistent mit dem beobachteten positiven Trend des NAO-Index bis Mitte der 1990er-Jahre (s. o.). Weiterhin unklar ist jedoch, ob dies auf den anthropogenen Klimawandel zurückzuführen ist (BACC II Author Team 2015:76). In diesem Zusammenhang konstatiert auch der IPCC in seinem 5. Sachstandsbericht, dass seit den 1970erJahren eine polwärtige Verlagerung der Zirkulation inklusive der Zugbahnen von Sturmzyklonen stattgefunden hat (IPCC 2013). Einige Autoren sind der Frage nachgegangen, ob die NAO durch ENSO (El Nino Southern Oscillation) beeinflusst wird (BACC II Author Team 2015, S. 75). Der direkte Einfluss wird jedoch aufgrund schwacher Korrelation als gering eingeschätzt. Ein nichtlinearer Einfluss der ENSO auf das europäische Klima wird aber für möglich gehalten. Bhend und Storch $(2008,2009)$ untersuchen den Einfluss des anthropogenen Klimawandels in Relation zur NAO auf Temperatur und Niederschlag im Ostseeraum und kommen zu dem Ergebnis, dass der anthropogene Einfluss einen großen Teil der beobachteten Änderungen erklären kann. Eine zufällige Schwankung ist hingegen als Ursache für die beobachteten Änderungen auszuschließen. Unter der Annahme, dass ein großer Teil der NAO nicht durch den anthropogenen Klimawandel beeinflusst wird, wurde die Analyse ohne Berücksichtigung der NAO wiederholt. Dabei zeigte sich, dass die Änderungen in Temperatur und Niederschlag weiterhin bestehen bleiben und als robust einzustufen sind, wohingegen jahreszeitliche Schwankungen und räumliche Ausprägungen von Temperatur und Niederschlag ohne Berücksichtigung der NAO gegenüber den beobachteten Werten unterschätzt wurden.

\subsubsection{Wind}

Lange Zeitreihen von Windmessungen können durch verschiedene Faktoren, insbesondere durch Stationsverlegungen, ein sich änderndes Messumfeld oder den Wechsel der Messinstrumente bzw. -methode, gestört werden (vgl. auch $\$ Abschn. 2.2). Auch die Detektion von Tiefdruckgebieten aus historischen Luftdruckfeldern wird durch eine sich mit der Zeit verändernde Datendichte beeinflusst. Aufgrund der zunächst geringen Datendichte können für die Vergangenheit auch nur entsprechend wenige Tiefdruckgebiete detektiert werden. Mit der Zeit hat die Datendichte fortlaufend zugenommen. Geht die zunehmende Datendichte in einen Langzeitdatensatz ein, werden hier allein aufgrund der im Vergleich zur Vergangenheit höheren Datendichte auch mehr Zyklonen detektiert. Diese veränderte Häufigkeit kann leicht fehlinterpretiert werden, ist jedoch nicht auf natürliche Schwankungen oder anthropogene Einflüsse, sondern auf Inhomogenitäten des Datensatzes zurückzuführen (BACC II Author Team 2015, S. 79).

Feser et al. (2015) dokumentieren Ergebnisse zur Änderung der Sturmklimas über dem Nordatlantik, der Ostsee und Nordwesteuropa, die in begutachteten Fachartikeln veröffentlicht wurden. Die wichtigste Erkenntnis dieser Dokumentation ist, dass Trends in der Sturmaktivität in einer bestimmten Region stark von dem jeweils zugrunde liegenden Untersuchungszeitraum abhängen (Feser et al. 2015, S. 350). Insgesamt zeigen die Ergebnisse der Langzeitanalysen von Sturmintensitäten über dem Nordatlantik und Nordwesteuropa starke dekadische Schwankungen, wodurch auch die Sensitivität der Trends hinsichtlich des Untersuchungszeitraums erklärt werden kann. Auch die größtenteils hohe Übereinstimmung der zeitlichen Abfolge von Sturmindizes mit den oben beschriebenen Schwankungen des NAO-Index unterstützt diese Annahme. So weist der Sturmindex nach Alexanderson et al. (2000) zu Beginn des 19. Jahrhunderts hohe Werte für Nordwesteuropa auf, gefolgt von einer Abnahme der Sturmindizes bis Mitte der 1960er-Jahre. Ab Mitte der 1960er-Jahre nimmt die Sturmintensität erneut zu, bis sie Mitte der 1990er-Jahre wieder abnimmt (Feser et al. 2015). Auch eine von Rosenhagen und Schatzmann (2011) im 1. HKB dokumentierte Analyse des geostrophischen Windes über der Deutschen Bucht zeigt im Zeitraum 1879-2005 keinen bzw. einen leicht negativen Trend (Rosenhagen 2008).

Viel mehr als durch einen linearen Trend wird die zeitliche Entwicklung der Sturmintensitäten durch starke Schwankungen geprägt. Zudem werden die hohen jährlichen Windgeschwindigkeiten der 1990er-Jahre in Relation zu länger zurückliegenden sturmreichen Jahren bewertet. Dabei zeigt sich, dass die hohen Windgeschwindigkeiten mit denen in den 1880er-Jahren und Mitte der 1920er-Jahre vergleichbar sind (Rosenhagen 2008), als ebenfalls hohe NAO-Indizes auftraten. Dies bestätigt auch eine Aktualisierung der Zeitreihe bis 2012 (Trusilova und Riecke 2015). 
Auch weitere Autoren bestätigen das Fehlen eines langfristigen Trends und die ausgeprägte Variabilität der Sturmaktivität (Kaas und Schmith 1996; WASA Group 1998; Alexandersson et al. 2000; Matulla et al. 2008). Auf der Basis eines räumlich und zeitlich homogenen Langzeitdatensatzes finden Weisse et al. (2005) eine Zunahme der mittleren Windgeschwindigkeit über der südlichen Nord- und Ostsee seit 1960. Auch Auswertungen im Rahmen des Norddeutschen Klimamonitors (Stand Oktober 2015², Meinke et al. 2014) auf Basis der Reanalysen coastDat1 (Weisse et al. 2009) und coastDat2 (Geyer 2014) zeigen im Zeitraum 1951-2010 in der Metropolregion Hamburg und Norddeutschland eine leichte Zunahme der mittleren jährlichen Windgeschwindigkeit.

Die Auswertung der Reanalysen coastDat1 und 2 zeigt weiterhin, dass diese Zunahme der mittleren Windgeschwindigkeit deutliche Unterschiede in den Jahreszeiten aufweist. Die deutlichsten Zunahmen der mittleren Windgeschwindigkeiten sind in der Metropolregion Hamburg und in Norddeutschland seit 1950 in den Wintermonaten zu verzeichnen, während die mittlere Windgeschwindigkeit in den Frühlings- und Sommermonaten nahezu unverändert bleibt. In ähnlicher Weise haben sich auch die Sturmintensitäten in der Hamburger Metropolregion und in Norddeutschland seit 1950 verändert. Wobei die Zunahme über der Nordsee stärker ausfällt als über der Ostsee und über Land. Bezüglich der Sturmhäufigkeit zeigen Feser et al. (2015), dass die meisten Studien seit den 1970er-Jahren bis etwa Mitte der 1990er-Jahre in einer Zunahme der Sturmhäufigkeit in der Region zwischen 55 und $60^{\circ} \mathrm{N}$ über dem Nordatlantik übereinstimmen.

Andere Studien, die auf der Basis von Proxys oder Messdaten einen längeren, etwa 100-jährigen Untersuchungszeitraum berücksichtigen, zeigen große dekadische Schwankungen, jedoch weder eine $\mathrm{Zu}$ - noch eine Abnahme der Sturmhäufigkeit über dem Nordatlantik. Kalnay et al. (1996) und Kistler et al. (2001) kommen durch die Auswertung von NCEP/NCAR-Reanalysen ab 1948 zu der Erkenntnis, dass die Häufigkeit von Tiefdruckgebieten unter $980 \mathrm{hPa}$ in den 1970er-Jahren im Winter ein Minimum aufweisen, in den darauffolgenden Dekaden zunehmen und in der letzten Dekade des 20. Jahrhunderts ein Maximum erreichen. Diese Entwicklung bestätigen auch Auswertungen im Rahmen des Norddeutschen Klimamonitors auf Basis der coastDat-1 und coastDat-2-Datensätze (Meinke et al. 2014). Die Ergebnisse zeigen für die letzten 60 Jahre im Mittel eine Zunahme der jährlichen Anzahl von Sturmtagen in Norddeutschland. Auch bei der Sturmhäufigkeit treten die größten Zunahmen im Winterhalbjahr auf, während in den Frühlings- und Sommermonaten wenige Änderungen in der Sturmhäufigkeit zu verzeichnen sind. Seit Beginn der 1980er-Jahre hat sich jedoch erneut ein negativer

2 Der Norddeutsche Klimamonitor wurde im Frühjahr 2017 aktualisiert. Dabei wurde der Datenbestand um den Zeitraum 2011 bis 2015 erweitert. Außerdem basieren Aussagen zur Klimaentwicklung nun nicht mehr auf linearen Trends zwischen 1951 bis 2010 sondern auf dem Vergleich der Zeitscheibe 1986-2015 mit 1961-1990. In diesem Kapitel werden Ergebnisse des Klimamonitors, Stand Oktober 2015 dokumentiert. Größtenteils weist die Klimaentwicklung in der aktualisierten Webseite weiterhin in dieselbe Richtung wie im Stand Oktober 2015. Bei Klimaelementen mit hoher Variabilität (z.B. Niederschlag) können sich manche Aussagen in diesem Kapitel jedoch von der inzwischen aktualisierten Webseite unterscheiden (Meinke 2017).
Trend in der Sturmhäufigkeit eingestellt. Dieser ist nicht konsistent mit den Trends der regionalen Klimaprojektionen (vgl. - Abschn. 2.4) und weist erneut auf den möglicherweise größeren dominanten Einfluss natürlicher Schwankungen (z. B. NAO) hin (Norddeutscher Klimamonitor, vgl. Meinke et al. 2014).

\subsubsection{Lufttemperatur}

Zahlreiche Studien belegen die Erwärmung in Nord- und Mitteleuropa (z. B. BACC II Author Team 2015). Beispielsweise liegt der lineare Trend der jährlichen mittleren Temperaturanomalien im Ostseeeinzugsgebiet südlich des 60. Längengrades im Zeitraum $1871-2011$ bei etwa $0,08^{\circ} \mathrm{C} /$ Dekade und erreicht ein Signifikanzniveau von 95 \% (BACC II Author Team 2015, S. 83). Diese Erwärmung entspricht in etwa dem Trend der globalen mittleren Lufttemperatur von etwa $0,09^{\circ} \mathrm{C} /$ Dekade im Zeitraum 1880-2012 (IPCC 2013, S. 187). Zudem weisen auch alle jahreszeitlichen Trends in dieser Region einen signifikanten positiven Trend in der bodennahen Lufttemperatur auf (BACC II Author Team 2015, S. 83).

Rosenhagen und Schatzmann (2011) dokumentieren im Rahmen des 1 . HKB ebenfalls einen positiven Trend der Jahresmitteltemperaturen anhand von Auswertungen der Station Hamburg-Fuhlsbüttel und stellen fest, dass die Intensität linearer Trends entscheidend von der Auswahl des Bezugszeitraums und dessen Länge abhängen. Um potenzielle Beschleunigungen im Anstieg der Jahresmitteltemperaturen mittels linearer Trends aufzuzeigen, unterteilen Schlünzen et al. (2009) lange Zeitreihen in mehrere Perioden, für die jeweils der lineare Trend bestimmt wird (s. auch KLIMZUG-NORD Verbund 2014). Für die Jahresmitteltemperatur in Hamburg-Fuhlsbüttel ergeben sich so folgende Trends, die als signifikant getestet wurden:

- 1891-2007: $0,07^{\circ} \mathrm{C} /$ Dekade

- 1948-2007: 0,19 ${ }^{\circ} \mathrm{C} /$ Dekade

- 1978-2007: 0,6 ${ }^{\circ} \mathrm{C} /$ Dekade

Trusilova und Riecke (2015) analysieren Gebietsmittel der Metropolregion Hamburg aus einem räumlich interpolierten Beobachtungsdatensatz hinsichtlich des zeitlichen Temperaturverlaufs von 1881 bis 2013. Für diesen Zeitraum zeigt sich eine Temperaturzunahme von etwa $1,4^{\circ} \mathrm{C}$. Diese entspricht einem linearen Trend von etwa $0,1^{\circ} \mathrm{C} /$ Dekade und liegt damit leicht über dem Trend von Rosenhagen und Schatzmann (2011) sowie Schlünzen et al. (2009) für den etwas kürzeren Zeitraum 1891-2007. Auch Auswertungen im Rahmen des Norddeutschen Klimamonitors (Stand Oktober 2015 ${ }^{3}$ ) zeigen für die Metropolregion Hamburg und Norddeutschland eine deutliche Erwärmung: Basierend auf den Datensätzen coastDat1 (Weisse et al. 2009) und coastDat2 (Geyer 2014) liegt dieser Trend der mittleren jährlichen Lufttemperatur in Norddeutschland und in der Metropolregion Hamburg im Zeitraum 1951-2010 bei $0,2{ }^{\circ} \mathrm{C} /$ Dekade. Aktualisierungen der Auswertungen des Norddeutschen Klimamonitors hinsichtlich einer Ergänzung der Datensätze bis zum Jahr 2015 bestätigen die oben genannten

3 Siehe Fußnote 2. 


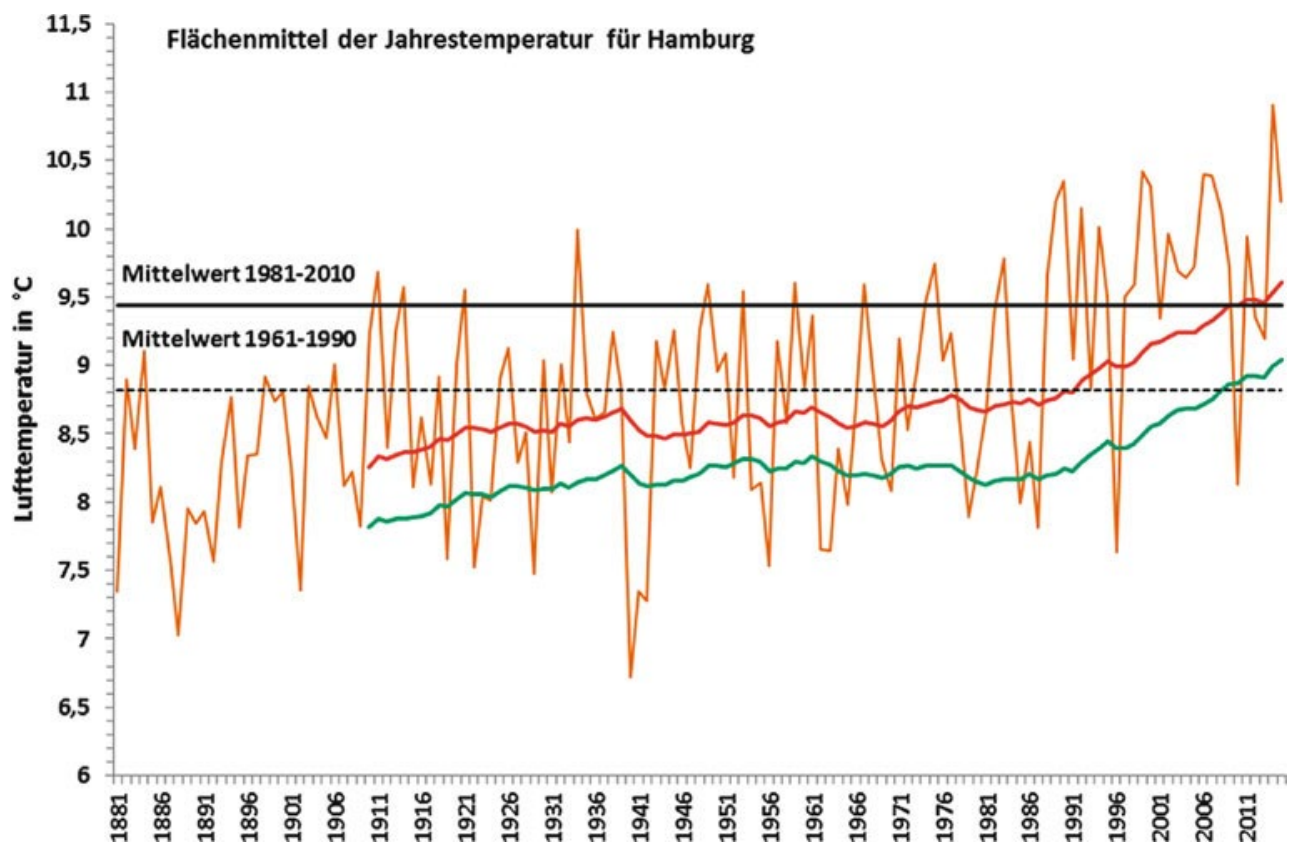

- Abb. 2.3 Entwicklung der Jahresmitteltemperaturen von 1881 bis 2015 für das Land Hamburg (braune Linie). Die dicke rote Linie stellt den langfristigen Trend als 30-jähriges gleitendes Mittel dar. Zum Vergleich ist das 30-jährige gleitende Mittel für Deutschland (dicke grüne Linie) gezeigt. Die schwarzen Linien kennzeichnen die Mittelwerte der Referenzperioden 1961-1990 (gestrichelt) und 1981-2010 (durchgezogen) für Hamburg. (Nach Trusilova und Riecke 2015, ergänzt durch Jahresmittel 2014 und 2015)
Erkenntnisse. Außerdem verdeutlicht die Aktualisierung, dass im Jahr 2014 die bisher größte jährliche Temperaturanomalie seit 1950 aufgetreten ist. Dies bestätigt auch die Ergänzung der Auswertung von Trusilova und Riecke (2015, vgl. • Abb. 2.3) für das Flächenmittel der Lufttemperatur. Am kältesten war hier mit 6,7 ${ }^{\circ} \mathrm{C}$ das Jahr 1940 (• Abb. 2.3).

Bei den zwölf Messstationen, die laut DWD in Norddeutschland größtmögliche Homogenität bzgl. der Messmethode und der Standorteigenschaften liefern und mindestens seit 1961 messen (vgl. Meinke et al. 2014), liegt der lineare Trend der 2-m-Lufttemperatur zwischen $0,2^{\circ} \mathrm{C} /$ Dekade (Brocken) und $0,28^{\circ} \mathrm{C} /$ Dekade (Rostock-Warnemünde). Alle Trends sind auf einem Signifikanzniveau von $95 \%$ statistisch signifikant (Norddeutscher Klimamonitor (Stand Oktober $2015^{4}$, Meinke et al. 2014)). Im jahreszeitlichen Vergleich dokumentiert das BACC II Author Team (2015) für den Zeitraum 1871-2011 in der südlichen Ostsee stärkste Trends im Frühjahr und Winter, während sie im Sommer am schwächsten ausfallen. Rosenhagen und Schatzmann (2011) zeigen im 1. HKB, dass auch die jahreszeitlichen Trends der mittleren Lufttemperatur stark vom Untersuchungszeitraum abhängen und beziehen sich dabei auf eine Untersuchung von Schlünzen et al. (2009). Für den Zeitraum 1948-2007 werden an der Station Hamburg-Fuhlsbüttel ähnlich hohe Trends im Frühjahr, im Sommer und im Winter festgestellt, während im Herbst ein deutlich geringerer Trend zu verzeichnen ist (Schlünzen et al. 2009). Dies bestätigen auch Auswertungen von Trusilova und Riecke (2015) in einem Vergleich der jahreszeitlichen Temperaturerhöhungen in den Zeiträumen 1981-2010 und 1961-1990 für die Flächenmitteltemperatur von Hamburg. Auch hier fiel die Erhöhung mit $0,1^{\circ} \mathrm{C}$ im Herbst am geringsten aus, während die Erwärmungen in den übrigen Jahreszeiten vergleichbar ausfielen $\left(+0,7^{\circ} \mathrm{C}\right.$ im Sommer, $+0,8^{\circ} \mathrm{C}$ im Winter und $+0,9^{\circ} \mathrm{C}$ im Frühjahr).

Auch Meinke et al. (2014) bestätigen diese Ergebnisse auf Basis von Stationsmessungen und Reanalysen für den Zeitraum

4 Siehe Fußnote 2.
1951-2010 in Norddeutschland und in der Metropolregion Hamburg. Beide Reanalysen coastDat 1 und coastDat 2 weisen ähnliche Trends im Frühjahr, Sommer und Winter von etwa $0,2^{\circ} \mathrm{C} /$ Dekade in Norddeutschland auf, während der Trend im Herbst mit $0,12^{\circ} \mathrm{C}$ (coastDat1) und $0,13^{\circ} \mathrm{C}$ (coastDat2) pro Dekade die niedrigsten Werte zeigt. Auch die Stationsdaten weisen vergleichbare lineare Trends innerhalb der Jahreszeiten auf, wobei die größten Trends jedoch an allen Stationen im Zeitraum 1951-2010 im Frühjahr liegen und Werte zwischen $0,33^{\circ} \mathrm{C}$ (Cuxhaven) und $0,39^{\circ} \mathrm{C}$ (Schwerin) pro Dekade aufweisen. Im Herbst weisen auch alle Stationsdaten die kleinsten Trends auf (Meinke et al. 2014). So liegen beispielsweise die herbstlichen Trends der Lufttemperaturen mit $0,12{ }^{\circ} \mathrm{C} /$ Dekade in Cuxhaven und $0,08^{\circ} \mathrm{C} /$ Dekade in Schwerin deutlich unter dem linearen Trend der Jahresmitteltemperaturen.

\subsubsection{Thermische Vegetationsindizes}

Das BACC II Author Team (2015) stellt für den Ostseeraum eine Verlängerung der thermischen Vegetationsperiode fest. Dabei handelt es sich um die Anzahl der Tage zwischen thermischem Vegetationsbeginn (erstes Aufkommen von mindestens 6 aufeinanderfolgenden Tagen mit einer Durchschnittstemperatur über $5{ }^{\circ} \mathrm{C}$ ) und thermischem Vegetationsende (erstes Aufkommen von mindestens 6 aufeinanderfolgenden Tagen mit einer Durchschnittstemperatur unter $5{ }^{\circ} \mathrm{C}$ im Winterhalbjahr). Chmielewski (2011) dokumentiert im 1. HKB für den Zeitraum 1961-2005 ebenfalls eine Verlängerung der thermischen Vegetationsperiode. Diese Verlängerung beträgt für Deutschland 25 Tage, wobei jedoch der Trend im Vegetationsbeginn (+19 Tage) wesentlich stärker ausfällt als beim Vegetationsende (+6 Tage). Der Norddeutsche Klimamonitor (Stand Oktober 2015) zeigt für Norddeutschland und für die Metropolregion Hamburg ebenfalls eine deutliche Verlängerung der thermischen Vegetationsperiode von etwa 3 Wochen seit 1950, wobei es in Norddeutschland deutliche regionale Unterschiede hinsichtlich des Ausmaßes der Verlängerung gibt (Meinke et al. 2014). Während sich die thermische Vegetationsperiode beispielsweise in Cuxhaven von 1952 bis 2010 um etwa 1,5 Monate 
(43 Tage) verlängert hat, fällt die Verlängerung der thermischen Vegetationsperiode auf dem Brocken mit zwei Wochen (14 Tage innerhalb des Zeitraums 1951-2010) deutlich kürzer aus.

Weiterhin bestätigen die Auswertungen im Norddeutschen Klimamonitor auch für Norddeutschland und für die Metropolregion Hamburg die Erkenntnis, dass die Verlängerung der thermischen Vegetationsperiode zum größten Teil auf eine deutliche Verfrühung des Vegetationsbeginns zurückzuführen ist. In diesem Zusammenhang kann die zeitliche Verlagerung des letzten Frosttages im Frühjahr relevant sein. Diese hat sich an allen im Norddeutschen Klimamonitor berücksichtigten Messstationen in Norddeutschland deutlich weniger verfrüht als der thermische Vegetationsbeginn. Aufgrund dieser unterschiedlichen Entwicklungen fallen Vegetationsbeginn und der letzte Frost an den jeweiligen Messstationen heute etwa 20-30 Tage weiter auseinander als vor 60 Jahren, wodurch das Spätfrostrisiko für die Vegetation höher geworden ist (Norddeutscher Klimamonitor (Stand Oktober $2015^{5}$ ), Meinke et al. 2014).

\subsubsection{Temperaturextreme}

Mit der Erwärmung hat sich auch die Häufigkeit von Extremereignissen geändert. Rosenhagen und Schatzmann (2011) dokumentieren im 1. HKB eine Zunahme der Überschreitungswahrscheinlichkeit der 90 \%-Perzentil-Schwelle der Lufttemperatur an verschiedenen deutschen Wetterstationen. Dabei zeigt sich auch in der Metropolregion Hamburg eine Häufigkeitszunahme höherer Temperaturen. Diese Trends sind - außer im Herbst - in allen Jahreszeiten im Zeitraum 1951-2000 statistisch signifikant. Zudem wird eine Studie von Riecke und Rosenhagen (2010) zitiert, in der anhand von Auswertungen der Station Hamburg-Fuhlsbüttel für den Zeitraum 1891-2007 eine Häufigkeitszunahme sowohl von Sommertagen (Temperaturmaximum $\geq 25^{\circ} \mathrm{C}$ ) als auch von heißen Tagen (Temperaturmaximum $\geq 30^{\circ} \mathrm{C}$ ) aufgezeigt wird. Eine entsprechende Auswertung bzgl. der Häufigkeitsänderungen von Eistagen (Temperaturmaximum $<0{ }^{\circ} \mathrm{C}$ ) zeigt dagegen erwartungsgemäß einen abnehmenden Trend. Trusilova und Riecke (2015) bestätigen diese Ergebnisse für Hamburg für den Zeitraum 1951-2013 und stellen fest, dass hier die Anzahl der Sommertage in den Jahren 2001-2013 durchgängig über dem langjährigen Mittel von 1961 bis 1990 lag. Auswertungen im Rahmen des Norddeutschen Klimamonitors (Stand $2015^{6}$ ) bestätigen ebenfalls die Aussagen bzgl. der Häufigkeitsveränderungen von Temperaturkenntagen in Norddeutschland für den Zeitraum 1951-2010. Dabei wird deutlich, dass sich die Häufigkeitszunahme von Sommertagen und heißen Tagen vor allem in den Sommermonaten vollzogen hat. Im Frühjahr und Herbst sind diese hohen Temperaturmaxima nach wie vor selten. Eine entsprechende Auswertung der Eistage zeigt die stärkste Häufigkeitsabnahme im Winter. Bezüglich der Häufigkeit von Frosttagen (Temperaturminimum $<0{ }^{\circ} \mathrm{C}$ ) ergeben sich jedoch auch im Frühjahr starke negative Trends im Zeitraum 1951-2010 (Meinke et al. 2014).

Untersuchungen von Imbery et al. (2015) zur jährlichen Häufigkeit einer 14-tägigen Hitzeperiode mit einem mittleren Tages-

5 Siehe Fußnote 2.

6 Siehe Fußnote 2. maximum der Lufttemperatur von mindestens $30^{\circ} \mathrm{C}$ für den Zeitraum 1950-2014 zeigen, dass in Hamburg solche Extremereignisse vor 1994 gar nicht auftraten, ab 1994 jedoch viermal. Insgesamt lässt sich festhalten, dass die bisherigen positiven Trends der Lufttemperatur und die bisherigen Trends der abgeleiteten Temperaturkenntage in der Metropolregion Hamburg seit 1980 mit den Trends der regionalen Klimaprojektionen konsistent sind (Norddeutscher Klimamonitor (Stand Oktober $2015^{7}$ ), vgl. Meinke et al. 2014). Schriebe man den bisherigen Trend seit 1981 gemäß dem Standarddatensatz coastDat-2 bis 2100 linear fort, läge die Änderung der durchschnittlichen Temperatur im Jahr bis Ende des 21. Jahrhunderts im Vergleich zur Referenzperiode (1961-1990) bei $+3{ }^{\circ} \mathrm{C}$. Diese Zunahme von $+3{ }^{\circ} \mathrm{C}$ ist mit den regionalen Klimaprojektionen konsistent: Aufgrund menschlicher Treibhausgasemissionen lassen die Projektionen eine Zunahme von etwa +1 bis $+5{ }^{\circ} \mathrm{C}$ bis 2100 plausibel erscheinen (vgl. Abschn. 2.4). Diese Konsistenz zwischen dem Trend bisheriger Änderungen und den Trends der regionalen Klimaprojektionen kann ein Indiz dafür sein, dass anthropogene Treibhausgasemissionen bereits zu der bisher stattgefundenen Erwärmung in der Hamburger Metropolregion beigetragen haben. In diesem Fall können die bisherigen Änderungen als Vorbote zu erwartender anthropogener Klimaänderungen gewertet werden (Meinke et al. 2014).

\subsubsection{Niederschlag}

Bezüglich der Niederschlagsentwicklung konstatiert das BACC 2 Author Team (2015) für den Ostseeraum eine größere Variabilität als bei der Temperaturentwicklung. Demnach variieren im Ostseeraum die Vorzeichen der Niederschlagstrends im 20. Jahrhundert sowohl regional als auch innerhalb der Jahreszeiten. In diesem Zusammenhang wird auch eine Studie von Casty et al. (2007) zitiert, in der für Europa hinsichtlich der mittleren Niederschlagshöhe große jährliche und dekadische Schwankungen festgestellt werden, jedoch kein Langzeittrend für den Zeitraum 1766-2000. Diese Ergebnisse legen eine regionalspezifischere Auswertung nahe, wobei auch hier methodische Hindernisse zu berücksichtigen sind. So sind Stationsmessungen insbesondere bei konvektiven Niederschlagsereignissen oft nicht räumlich repräsentativ und weisen systematische Fehler bei der Messung des Niederschlags auf (Rosenhagen und Schatzmann 2011). Auch Reanalysen des Niederschlags sind mit Unsicherheiten behaftet, da z. B. die räumliche Auflösung der Modelle oft noch zu niedrig ist, um konvektive Prozesse realitätsnah abzubilden. Rosenhagen und Schatzmann (2011) dokumentieren eine $\mathrm{Zu}$ nahme der jährlichen Niederschlagshöhe in Deutschland während des Zeitraums 1900-2007 von etwa 750 auf ca. $800 \mathrm{~mm}$. Gleichzeitig wird jedoch eine hohe Variabilität festgestellt, wodurch der Signifikanztest verfehlt wird. Auswertungen der Niederschlagszeitreihe von Hamburg-Fuhlsbüttel (Schlünzen et al. 2009) bestätigen auch für Hamburg eine Zunahme der jährlichen Niederschlagshöhe von 1891 bis 2007 von $8 \mathrm{~mm} /$ Dekade (Rosenhagen und Schatzmann 2011). Auch Trusilova und Riecke (2015) zeigen für den Zeitraum 1881-2013 einen

7 Siehe Fußnote 2. 


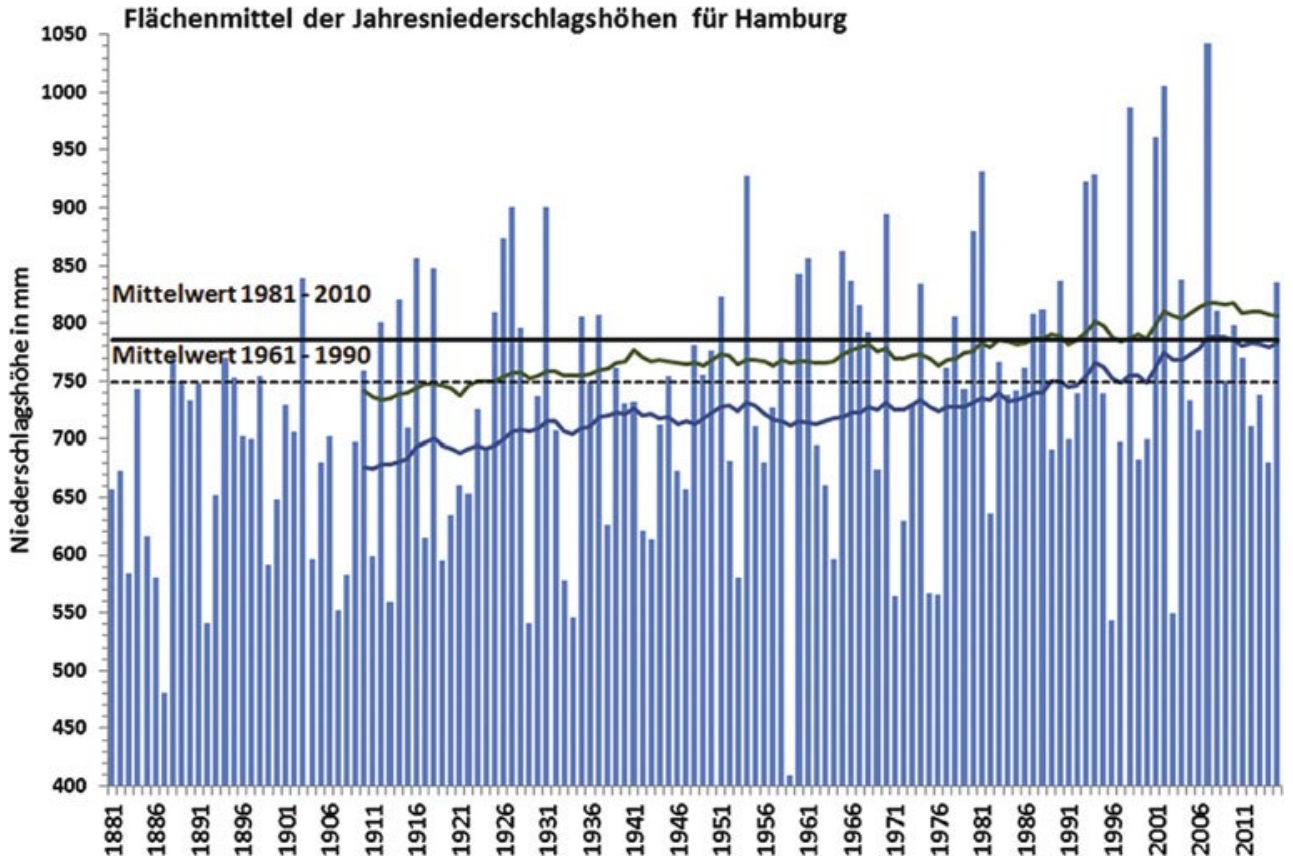

- Abb. 2.4 Entwicklung der Gebietsmittel der Jahresniederschlagshöhen von 1881 bis 2015 für das Land Hamburg (blaue Säulen). Die dicke blaue Linie stellt den langfristigen Trend als 30-jähriges gleitendes Mittel dar. Zum Vergleich ist das 30-jährige gleitende Mittel für Deutschland (dicke grüne Linie) gezeigt. Die schwarzen Linien kennzeichnen den Mittelwert der Referenzperioden 1961-1990 (gestrichelt) und 1981-2010 (durchgezogen) für Hamburg. (Nach Trusilova und Riecke 2015, ergänzt durch Jahressummen 2014 und 2015) ansteigenden Trend der Niederschlagshöhen von ca. $120 \mathrm{~mm}$ in Hamburg (entsprechend 0,9 mm/Dekade, - Abb. 2.4).

Auswertungen von Stationsdaten (Helgoland, Cuxhaven und Schwerin) bestätigen eine mittlere Niederschlagszunahme über die letzten Jahrzehnte in der Metropolregion Hamburg (Norddeutscher Klimamonitor (Stand Oktober $2015^{8}$ ), Meinke et al. 2014). Für ganz Norddeutschland können jedoch bzgl. der Niederschlagsentwicklung von 1951 bis 2010 keine einheitlichen Ergebnisse abgeleitet werden. Für den genannten Zeitraum existieren in Norddeutschland sechs Stationen mit hinreichend wenigen Fehlwerten bei der Niederschlagsmessung, wobei sich diese zumeist im Osten Norddeutschlands befinden (Brocken, Potsdam, Waren, Marnitz, Rostock und Arkona). Trotz dieser regionalen Häufung von langfristigen Niederschlagsmessungen weisen die Stationsdaten jährliche Niederschlagstrends mit unterschiedlichen Vorzeichen auf. So haben auf dem Brocken, in Waren und in Rostock die jährlichen Niederschlagssummen von 1951 bis 2010 zugenommen, während sie in Potsdam, Marnitz und Arkona im selben Zeitraum abnahmen. Auch die Reanalysen coastDat 1 und coastDat2 weisen bei der Auswertung der Gebietsmittel ganz Norddeutschlands hinsichtlich der Entwicklung der jährlichen Niederschlagshöhe unterschiedliche Vorzeichen im Zeitraum 1951-2010 auf. In der räumlichen Ausprägung der jährlichen Niederschlagshöhe innerhalb Norddeutschlands lassen sich jedoch auch Übereinstimmungen beider Datensätze erkennen. So zeigen sowohl coastDat1 als auch coastDat2 Zunahmen der jährlichen Niederschlagshöhen an der deutschen Nordseeküste und über der Deutschen Bucht. Niederschlagsabnahmen zeigen beide Datensätze im südlichen Mecklenburg-Vorpommern und im nördlichen Brandenburg.

Im jahreszeitlichen Vergleich dokumentieren Rosenhagen und Schatzmann (2011) außer im Sommer einen leicht zuneh-

8 Siehe Fußnote 2. menden Trend der jahreszeitlichen Niederschlagshöhen in der Metropolregion Hamburg. Dies gilt sowohl für den Zeitraum 1901-2000 als auch für den kürzeren Zeitabschnitt 1971-2000. Auch die Auswertungen der Niederschlagsmessungen an der Messstation Hamburg-Fuhlsbüttel bestätigen diese Ergebnisse, wobei der positive Trend im Frühjahr am schwächsten ausfällt. Auswertungen im Norddeutschen Klimamonitor (Stand Oktober $2015^{9}$, Meinke et al. 2014) bestätigen im Wesentlichen diese Ergebnisse für Norddeutschland und die Metropolregion Hamburg. Im Zeitraum 1951-2010 weisen alle sechs Stationsdaten, die diesen Zeitraum abdecken, im Frühjahr, Herbst und Winter Zunahmen der jahreszeitlichen Niederschlagshöhen auf. Dies zeigt sich ebenfalls bei den Zeitreihen der Gebietsmittel für Norddeutschland und für die Metropolregion Hamburg, basierend auf den Datensätzen coastDat1 und coastDat2. Im Sommer stimmen beide coastDat-Datensätze für diesen Zeitraum in einer Abnahme der saisonalen Niederschlagshöhen überein. Auch an den Messstationen weisen die Niederschlagstrends im Sommer überwiegend auf eine Abnahme der Niederschlagshöhe hin. Trusilova und Riecke (2015) finden in Hamburg durch Vergleich von zwei 30-jährigen Zeitscheiben (1961-1990 und 1981-2010) für alle Jahreszeiten Zunahmen in den Niederschlagshöhen. Diese fallen im Winter und im Herbst am deutlichsten aus. Dies zeigt, dass neben der Auswahl des Untersuchungszeitraums auch die Auswertemethode Einfluss auf das Ergebnis hat. Dies verdeutlicht ein Vergleich der Methoden im Norddeutschen Klimamonitor hinsichtlich der zeitlichen Entwicklung des Sommerniederschlags. Werden die beiden Zeitscheiben 1961-1990 und 1981-2010 verglichen, zeigen die meisten Datensätze für die Metropolregion Hamburg eine Zunahme des Sommerniederschlags. Werden jedoch lineare Trends von 1951 bis 2010 ausgewertet, ergeben die Datensätze, die den gesamten Zeitraum

9 Siehe Fußnote 2. 
abdecken, eine Abnahme. Insgesamt lässt sich zusammenfassen, dass bei der langzeitlichen Entwicklung der jahreszeitlichen Niederschlagshöhen eine starke Abhängigkeit der Trends vom jeweils zugrunde liegenden Untersuchungszeitraum gegeben ist. Dies wiederum ist bei der starken natürlichen Variabilität des Niederschlags zu erwarten.

Rosenhagen und Schatzmann (2011) zitieren im 1. HKB eine Studie von Schlünzen et al. (2009), in der die Auswertungen von neun Stationen während des Zeitraums 1978-2007 eine zunehmende Tendenz bei der Anzahl von regenreichen Tagen $(10 \mathrm{~mm} /$ Tag) im Hamburger Raum erkennen lassen. Außerdem wird eine Studie von Hoffmann (2009) zitiert, in der belegt wird, dass zwar die Anzahl der Niederschlagstage seit 1890 etwa gleich geblieben ist, die Anzahl von Tagen mit wenig Niederschlag jedoch abgenommen und die Häufigkeit von Tagen mit starkem Niederschlag ( $\geq 10 \mathrm{~mm} / \mathrm{Tag}$ ) um etwa $24 \%$ zugenommen hat. Daraus wird geschlossen, dass die Zunahme der jährlichen Niederschlagshöhe vor allem auf die Zunahme von Tagen mit stärkerem Niederschlag zurückzuführen ist. Insgesamt dokumentieren Rosenhagen und Schatzmann (2011) jedoch keine statistisch signifikanten Trends bei der Häufigkeit von Starkniederschlagsereignissen. Auch Auswertungen im Norddeutschen Klimamonitor (Stand Oktober $2015^{10}$, Meinke et al. 2014) stellen eine Häufigkeitszunahme von regenreichen Tagen ( $\geq 10 \mathrm{~mm} / \mathrm{Tag}$ ) pro Jahr in Norddeutschland während des Zeitraums 1951-2010 fest. Dies zeigen sowohl die Auswertungen von Stationsdaten als auch die beiden Reanalysen coastDat1 und coastDat2, wobei auch diese Trends nicht signifikant sind. Im jahreszeitlichen Vergleich ist diese Häufigkeitszunahme im Winter am stärksten. Im Sommer ist die Datenlage bzgl. der Häufigkeitsänderungen von regenreichen Tagen $(\geq 10 \mathrm{~mm} /$ Tag) sowohl bei den Stationsdaten als auch bei den Reanalysen coastDat1 und 2 uneinheitlich, da es auch Hinweise auf Häufigkeitsabnahmen von regenreichen Tagen ( $\geq 10 \mathrm{~mm} / \mathrm{Tag}$ ) gibt. Insgesamt hat sich die Anzahl der sommerlichen Niederschlagstage von 1951 bis 2010 leicht reduziert, sodass es scheint, als sei die Abnahme der sommerlichen Niederschlagshöhe in manchen Regionen Norddeutschlands eher einer abnehmenden Häufigkeit von Niederschlagstagen insgesamt als einer Häufigkeitsänderung von regenreichen Tagen ( $\geq 10 \mathrm{~mm} / \mathrm{Tag}$ ) zuzuordnen. Im Herbst hingegen nehmen die Häufigkeiten von regenreichen Tagen etwa gleich stark zu, sodass die Niederschlagszunahme im Herbst vor allem mit einer Zunahme regenreicher Tage einherzugehen scheint.

Weiterhin zeigt sich, dass die Dauer der längsten Trockenperiode in Norddeutschland von 1951-2010 vor allem im Sommer und im Frühjahr zugenommen hat. Die Anzahl der Trockenperioden hat sich im Jahresverlauf jedoch nicht nennenswert verändert (Norddeutscher Klimamonitor (Stand 2015 ${ }^{11}$, Meinke et al. 2014)).

10 Siehe Fußnote 2.

11 Siehe Fußnote 2.

\subsection{Mögliche Änderungen des Klimas im 21. Jahrhundert}

\subsubsection{Einleitung: Klimaprojektionen für das 21. Jahrhundert}

Die zukünftige Entwicklung des Klimas im 21. Jahrhundert wird zum einen durch die interne Variabilität des Klimas, zum anderen durch externe Faktoren bestimmt. Die interne Variabilität des Klimas entsteht durch natürliche Prozesse innerhalb des Klimasystems und durch Wechselwirkungen zwischen seinen Komponenten. Sie führt zu stochastischen Schwankungen der Klimaparameter auf unterschiedlichen Zeitskalen. Zum Beispiel entstehen bei Prozessen zum Austausch von Wärme zwischen Atmosphäre und Ozean natürliche Klimaschwankungen, die sich auch über mehrere Jahrzehnte bemerkbar machen können. Bei den externen Faktoren werden natürliche und anthropogene unterschieden. Natürliche externe Faktoren sind z. B. vulkanische Aktivität oder Schwankungen der Sonneneinstrahlung. Emissionen von Treibhausgasen und Aerosolen spielen als externer anthropogener Faktor eine wesentliche Rolle für das Klima im 21. Jahrhundert. Um abzuschätzen, wie sich bei weiteren anthropogenen Emissionen das Klima in Zukunft entwickeln kann, werden Klimamodelle verwendet. Dazu werden Emissionsszenarien für das 21. Jahrhundert entwickelt, die auf verschiedenen Annahmen z. B. zur Entwicklung der Bevölkerung, der menschlichen Kultur, der Technologie und der Wirtschaft beruhen. Mit Klimamodellen werden die Auswirkungen der veränderten atmosphärischen Zusammensetzung auf das Klimasystem der Erde simuliert. Um die regional unterschiedlichen Ausprägungen der Klimaänderungen genauer zu untersuchen, werden die Simulationen der globalen Modelle mit regionalen Klimamodellen räumlich verfeinert. Eine ausführlichere $\mathrm{Be}$ schreibung der Methodik von Klimaprojektionen findet sich in Box „Methodik Klimaprojektionen“. Für Europa und Deutschland wurden zahlreiche regionale Klimaprojektionen für das 21. Jahrhundert erstellt und in verschiedenen Projekten und Studien für die Metropolregion Hamburg ausgewertet. Nachfolgend wird der aktuelle Kenntnisstand zu möglichen Klimaänderungen in der Region im 21. Jahrhundert dargestellt.

\section{Methodik Klimaprojektionen}

Emissionsszenarien

Um die zukünftig möglichen anthropogenen Emissionen von klimawirksamen Substanzen abzuschätzen, werden Emissionsszenarien für das 21. Jahrhundert entwickelt. Zur Erstellung der im „Special Report on Emission Scenarios (SRES)“ publizierten Szenarien von Nakicenovic und Swart (2000) wurden zunächst in sich konsistente Annahmen zur globalen demographischen, sozioökonomischen und technologischen Entwicklung in der Zukunft getroffen und daraus die Entwicklungen der anthropogenen Emissionen abgeleitet (die SRES-Szenarien werden im 1. HKB näher erläutert). Daraus werden die atmosphärischen Konzentrationen von Treibhausgasen und Aerosolen berechnet und deren Wirkung auf die Strahlungsbilanz der Erde mit Klimamodellen berechnet. Um 2010 wurde ein weiteres Konzept für die Erstellung globaler Szenarien entwickelt, das auf "repräsentativen Konzentrationspfaden“ („representative concentration pathways", RCPs) beruht (Moss et al. 2010). Sie werden durch den Strahlungsantrieb zum Ende des 21. Jahrhunderts definiert und repräsentieren 
verschiedene Entwicklungspfade der Treibhausgaskonzentrationen und zugehöriger Emissionen. So führt beispielsweise der Konzentrationspfad des RCP4.5 zum Ende des 21. Jahrhunderts zu einem Strahlungsantrieb von etwa 4,5 W/ $\mathrm{m} 2$. Diese physikalischen Schwellenwerte können durch verschiedene sozioökonomische Entwicklungen erreicht werden, die z. B. auch klimapolitische Maßnahmen berücksichtigen. Der Konzentrationspfad des RCP2.6 beinhaltet sehr ambitionierte Maßnahmen zur Reduktion von Treibhausgasemissionen und zum Ende des 21. Jahrhunderts sogar "negative Emissionen" (Fuss et al. 2014). Er führt zum Strahlungsantrieb von etwa $3 \mathrm{~W} / \mathrm{m} 2$ um 2040 und geht dann gegen Ende des 21. Jahrhunderts auf einen Wert von 2,6 W/m2 zurück. RCP2.6 repräsentiert damit den im Vergleich zu allen RCP- und SRES-Szenarien geringsten Strahlungsantrieb. Mit RCP8.5 dagegen wird ein kontinuierlicher Anstieg der Treibhausgasemissionen beschrieben und zum Ende des 21. Jahrhunderts ein Strahlungsantrieb von 8,5 W/m2 erreicht. Gegenwärtig befinden wir uns auf dem Pfad des RCP8.5 (Fuss et al. 2014). In - Abb. 2.5 sind die anthropogenen Strahlungsantriebe auf Basis der SRES- und RCP-Szenarien gemeinsam dargestellt.

\section{Erdsystemmodelle}

Klimamodelle sind dreidimensionale Zirkulationsmodelle der Atmosphäre, meist gekoppelt mit Modellen des Ozeans. Die gekoppelten AtmosphärenOzean-Modelle wurden zu Erdsystemmodellen weiterentwickelt, die auch Modelle der Biosphäre, des Meereises und von Eisschilden sowie Aerosole und chemische Prozesse in der Atmosphäre beinhalten. Damit werden auch biogeochemische Kreisläufe wie z. B. der Kohlenstoff-, der Stickstoff- und der Schwefelkreislauf abgebildet. Die interaktive Kopplung biogeochemischer Kreisläufe mit physikalischen Prozessen im Klimasystem ist eine wesentliche Neuerung von Erdsystemmodellen. Damit können anthropogene Emissionen direkt im Modell vorgegeben, die Verteilung des zusätzlichen Kohlendioxids in Atmosphäre, Ozean und Biosphäre simuliert und dabei auch Rückkopplungsprozesse im Klimasystem erfasst werden. Die Weiterentwicklung der Klimamodelle hat das Verständnis von Prozessen im Klimasystem fortlaufend erweitert. Weltweit wurden und werden an vielen Zentren Klimamodelle entwickelt. Die Modelle reagieren z. T. unterschiedlich empfindlich auf die veränderten Treibhausgaskonzentrationen in der Atmosphäre. Sie unterscheiden sich z. B. in numerischen Lösungsmethoden und physikalischen Parametrisierungen sowie in der Repräsentierung und Kopplung von Teilsystemen und Prozessen im Klimasystem. Dabei kann nicht gesagt werden, welches der Modelle richtig oder besser ist als andere. Es kann zwar untersucht werden, wie gut die Modelle das historische Klima und beobachtete Prozesse im Klimasystem wiedergeben, doch das lässt nicht automatisch darauf schließen, ob diese Modelle auch das zukünftige Klima in gleicher Güte abbilden.

\section{Ensemble-Simulationen}

Um die unterschiedlichen Klimasensitivitäten der Klimamodelle zu berücksichtigen, werden nach aktuellem Kenntnisstand Klimaprojektionen mit vielen Klimamodellen in Multi-Modell-Ensembles auf der Basis mehrerer Emissionsszenarien erstellt. Um den Einfluss der internen Variabilität auf die zukünftige Klimaentwicklung unter veränderten äußeren Randbedingungen zu berücksichtigen, werden die Simulationen für heutiges und zukünftiges Klima auf der Basis ein und desselben Emissionsszenarios mehrmals durchgeführt. Die Simulationen unterscheiden sich im Ausgangszustand des Klimasystems und bilden jeweils unterschiedliche zeitliche Verläufe der Klimaentwicklung ab. Insgesamt ergeben sich aus den Ensemble-Simulationen Bandbreiten möglicher Reaktionen des Klimasystems für jedes der betrachteten Emissionsszenarien. Zur Durchführung von Multi-Modell-Ensemble-Simulationen sind koordinierte Experimente notwendig, um die Vergleichbarkeit und einen geeigneten Austausch von Daten zu gewährleisten.

So wurden vom World Climate Research Program (WCRP) internationale Modellvergleichsprojekte initiiert. Beim „Coupled Model Intercomparison Project Phase 3" (CMIP3, Meehl et al. 2007) wurden koordinierte Simulationen mit gekoppelten Atmosphäre-Ozean-Modellen auf der Basis verschiedener SRES-Emissionsszenarien erstellt. Im CMIP5 (Taylor et al. 2012) wurden Simulationen mit gekoppelten Atmosphäre-Ozean-Modellen und Erdsystemmodellen auf der Basis verschiedener RCPs erstellt. Die Daten werden in standardisierter Form weltweit über die Earth System Grid Federation (ESGF) bereitgestellt. Vergleiche zu den Ergebnissen der Klimaprojektionen auf Basis

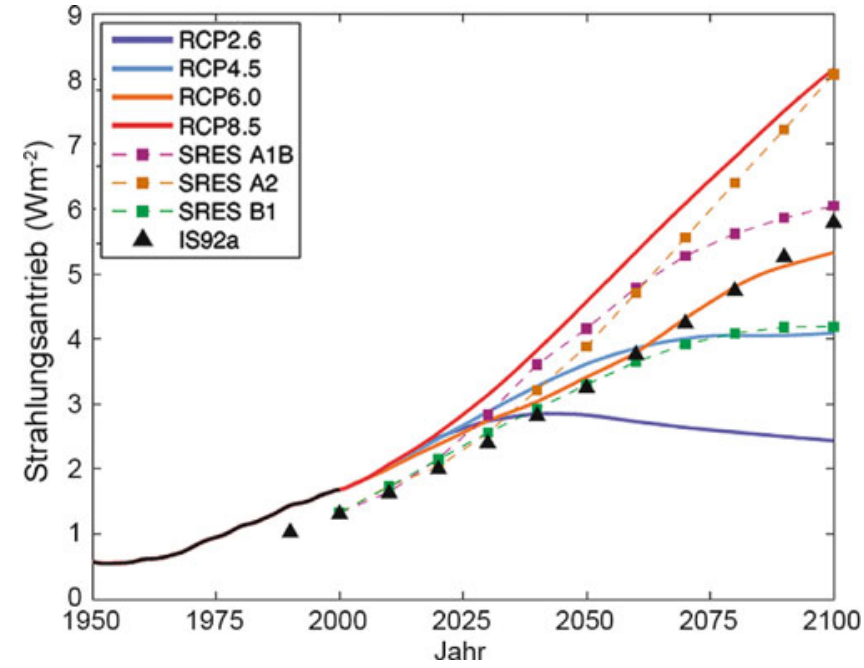

- Abb. 2.5 Historischer und projizierter anthropogener Strahlungsantrieb (W m-2) für 1950-2100, relativ zum vorindustriellen Wert um 1765. Es sind die Werte auf Basis der SRES-Emissionsszenarien im Vergleich zu den RCPs und im Vergleich zu einem „Business as usual“-Szenario IS92a dargestellt. (IPCC 2013, Fig. 1.15)

von CMIP3 und CMIP5 finden sich z. B. in Rogelj et al. (2012) sowie Knutti und Sedláček (2013). Am internationalen Modellvergleichsprojekt „Coupled Model Intercomparison Project - Phase 5“ (CMIP5; Taylor et al. 2012) waren etwa 20 Modellierungsgruppen mit mehr als 40 Modellen beteiligt - fast doppelt so viele wie beim Vorgängerprojekt CMIP3 (Meehl et al. 2007). Die Simulationen des CMIP5 "Multi-Modell-Ensemble" bilden die Basis für den 5. Sachstandsbericht des IPCC (2013). Einerseits bestätigen und bekräftigen die Ergebnisse des 5. Sachstandsberichtes die Ergebnisse des Vorgängerberichtes. Zugleich konnte das Verständnis zu den Prozessen im Klimasystem deutlich erweitert werden.

Die Regionalisierung globaler Klimaprojektionen ermöglicht die Untersuchung der Auswirkungen globaler Klimaänderungen auf einzelne Regionen. Mit der dynamischen Regionalisierungsmethode wird ein dreidimensionaler Ausschnitt des Klimasystems mit einem regionalen Klimamodell simuliert. Statistische Verfahren beruhen auf empirischen Zusammenhängen zwischen der beobachteten großräumigen Zirkulation in der Atmosphäre und dem lokalen Wettergeschehen an Messstationen. Mit regionalen Klimamodellen werden zunehmend international koordinierte Multi-Modell-Ensembles erstellt. Zum einen werden Simulationen verschiedener Globalmodelle regionalisiert, da unterschiedliche abgebildete großskalige Strömungsmuster auch in den regionalen Modellen übernommen werden. Zum anderen unterscheiden sich auch Regionalmodelle durch physikalische Parametrisierungen und numerische Lösungsmethoden. Deshalb werden Simulationen verschiedener Globalmodelle mit verschiedenen regionalen Modellen kombiniert. Solche Multi-Global-/Regionalmodell-Kombinationen wurden für Europa zunächst im Rahmen der EUProjekte PRUDENCE (Christensen et al. 2002) und ENSEMBLES (van der Linden und Mitchell 2009) auf der Basis der SRES-Szenarien erstellt. Im Rahmen der internationalen Initiative EURO-CORDEX wurden und werden koordinierte Simulationen mit einer horizontalen Auflösung von 12 km für Europa, basierend auf den RCPs, durchgeführt (Jacob et al. 2014).

\subsubsection{Projizierte Klimaänderungen in der Metropolregion Hamburg im 21. Jahrhundert}

Insgesamt wurden für Europa und Deutschland zahlreiche regionale Klimaprojektionen für das 21. Jahrhundert erstellt (z. B. Christensen et al. 2002; Spekat und Kreienkamp 2007; Orlowsky 
et al. 2008; Hollweg et al. 2008; van der Linden und Mitchell 2009; Kreienkamp et al. 2011; Jacob et al. 2008, 2012, 2014; Wagner et al. 2013; Rechid et al. 2014a, 2014b, 2014c, 2014d). Sie unterscheiden sich in den verwendeten globalen Klimamodellen und Regionalisierungsmethoden, in den zugrunde liegenden Emissionsszenarien und in den Startbedingungen der Simulationen. Aus den Modellsimulationen können Bandbreiten möglicher zukünftiger Änderungen der verschiedenen Klimaelemente und den daraus abgeleiteten Größen in Norddeutschland und der Metropolregion Hamburg ausgewertet werden (z. B. Meinke und Gerstner 2009; Meinke et al. 2011; Moseley et al. 2012; Jacob et al. 2012; Linde et al. 2014a, 2014b; Schötter et al. 2012; Rechid et al. 2014a, 2014b, 2014c, 2014d). Die Ergebnisse des 1. HKB wurden aus regionalen Klimaprojektionen für verschiedene SRES-Emissionsszenarien abgeleitet. Diese wurden mit verschiedenen dynamischen und statistischen Methoden auf der Grundlage globaler Projektionen des gekoppelten Modellsystems ECHAM5-MPIOM erstellt. In dem Verbundprojekt KLIMZUG-NORD („Strategische Anpassungsansätze zum Klimawandel in der Metropolregion Hamburg") wurden weitere Simulationen des regionalen Klimamodells CLM (Hollweg et al. 2008) und des regionalen Klimamodells REMO (Rechid et al. 2014a) verwendet; auch sie basieren alle auf den globalen Projektionen des gekoppelten Modellsystems ECHAM5-MPIOM sowie den SRES-Szenarien A2, A1B und B1.

Im Mai 2015 wurde der 2. Klimabericht für das Einzugsgebiet der Ostsee veröffentlicht (BACC II Author Team 2015). Die darin zusammengeführten Studien und Informationen zu möglichen Klimaänderungen in der Region, die am Rand auch das Gebiet der Metropolregion Hamburg einschließen, basieren zum großen Teil auf den regionalen Klimaprojektionen des ENSEMBLES-Projektes in $25 \mathrm{~km}$ horizontaler Auflösung für Europa auf Basis von globalen Simulationen des CMIP3 für das SRES-Emissionsszenario A1B. Im Rahmen der internationalen CORDEX-Initiative (Giorgi et al. 2009) werden koordinierte regionale Simulationen auf Basis der repräsentativen Konzentrationspfade RCPs und globaler Simulationen des CMIP5 mit einer horizontalen Auflösung von etwa $50 \mathrm{~km}$ auch für Europa (EUR-44) durchgeführt (z. B. Kotlarski et al. 2014). Im Rahmen der Initiative EURO-CORDEX wurden zudem hochaufgelöste Klimaänderungssimulationen auf Rastern mit Kantenlängen von 12 km (EUR-11) auf Basis der RCPs und globaler Simulationen des CMIP5 erstellt (Jacob et al. 2014; Kotlarski et al. 2014; Vautard et al. 2013). Sie erweitern die aktuelle Datenbasis zu projizierten Klimaänderungen für die Metropolregion Hamburg im 21. Jahrhundert.

Der Norddeutsche Klimaatlas integriert die regionalen Klimaprojektionen aus den bisherigen (Stand Oktober 2015) europäischen Multi-Model-Ensemble-Initiativen PRUDENCE, ENSEMBLES und EURO-CORDEX sowie aus nationalen Initiativen zur Erstellung regionaler Klimaprojektionen auf Basis der SRES- (A2, A1B, B2 und B1) und RCP-Szenarien (EUR-11 und EUR-44) mit dem Ziel, Spannbreiten möglicher künftiger Klimaänderungssignale von Wetterelementen und abgeleiteten Größen für Norddeutschland und Teilregionen darzustellen (siehe Datengrundlage der Webseite Norddeutscher Klimaatlas). Die Datenbasis bilden derzeit 123 regionale Klimaprojektionen, die
Norddeutschland räumlich abdecken und öffentlich verfügbar sind. Neben den Auswertungen für Norddeutschland werden für alle norddeutschen Bundesländer und verschiedene Naturräume wie auch für die Metropolregion Hamburg Spannbreiten möglicher Änderungen angegeben. Die Auswahl der jeweiligen Simulationen innerhalb der Emissionsszenarien (SRES) bzw. innerhalb der repräsentativen Konzentrationspfade (RCP) erfolgt, indem für jedes Klimaelement die regionale Klimaprojektion mit dem jeweils kleinsten und dem jeweils größten Änderungssignal für das jeweilige Zeitfenster ausgewählt wird. Auf diese Weise werden Spannbreiten möglicher künftiger Klimaänderungen in Norddeutschland auf Basis von RCP- und SRES-Szenarien abgeleitet.

Im Folgenden wird ein Überblick zu Studien projizierter Änderungen atmosphärischer Klimaparameter auf Basis der oben aufgeführten Klimaprojektionen gegeben, die nach dem 1. HKB erschienen sind. Insgesamt sind bei der Synthese und Interpretation von veröffentlichten Zahlen zu projizierten Klimaänderungen immer die verwendeten Datengrundlagen und Auswertemethoden, die dabei verwendeten zeitlichen und räumlichen Kennwerte sowie die betrachteten Zeitperioden zu beachten.

\subsubsection{Lufttemperatur}

Die projizierten Änderungen der bodennahen Lufttemperatur für Simulationen der regionalen Klimamodelle CLM und REMO wurden für die Metropolregion Hamburg, für Niedersachsen und Deutschland konsistent ausgewertet (Jacob et al. 2012; Moseley et al. 2012; Rechid et al. 2014a, 2014b, 2014c, 2014d). Die Simulationen und Auswertemethoden sind ausführlich in Rechid et al. (2014a) beschrieben. Dort sind kontinuierliche Zeitreihen sowie die beiden Zeitscheiben 2036-2065 und 2071-2100 im Vergleich zum Zeitraum 1971-2000 ausgewertet. Die verschiedenen Realisierungen für ein Emissionsszenario zeigen Unterschiede der klimatologischen Jahresmitteltemperatur von bis $\mathrm{zu} 1^{\circ} \mathrm{C}$. Das verdeutlicht die durch natürliche interne Klimavariabilität verursachte Schwankungsbreite, wie sie in der Modellkette Globalmodell-Regionalmodell abgebildet wird. In allen Simulationen steigt die mittlere Jahrestemperatur für das Gebietsmittel der Metropolregion Hamburg an, zur Mitte des 21. Jahrhunderts um etwa $1-2{ }^{\circ} \mathrm{C}$ und zum Ende des Jahrhunderts um etwa 2 bis mehr als $3{ }^{\circ} \mathrm{C}$. Dabei ist die Erwärmung im Winter mit $2-4^{\circ} \mathrm{C}$ etwas stärker als im Sommer mit $1-3^{\circ} \mathrm{C}$. Werden allerdings regionale Klimaprojektionen auf der Basis von Simulationen mit verschiedenen Globalmodellen betrachtet - z. B. in BACC II Author Team (2015) im Vergleich zu 1961-1990 und in Jacob et al. (2014) im Vergleich zu 1971-2000 - , dann reicht die projizierte Temperaturänderung im Sommer von etwa 1 bis $4{ }^{\circ} \mathrm{C}$.

In $-\mathrm{Abb}$. 2.6 sind die auf der Basis der EURO-CORDEX-Simulationen EUR-11 (Jacob et al. 2014) projizierten Änderungen der jährlichen bodennahen Lufttemperatur im Vergleich zum Zeitraum 1971-2000 als gleitendes 30-Jahres-Mittel (jeweils abgebildet auf das 30. Jahr) für das Gebietsmittel der Metropolregion Hamburg dargestellt. Die gesamte Bandbreite der simulierten jährlichen Temperaturänderungen mit dem EURO-CORDEXEnsemble im Vergleich zu 1971-2000 beträgt für die Zeitperiode 2036-2065 etwa $1-2,5^{\circ} \mathrm{C}$ und für $2071-2100$ etwa $1-4{ }^{\circ} \mathrm{C}$. Die Ergebnisse für Sommer, Herbst und Winter sehen sehr ähn- 


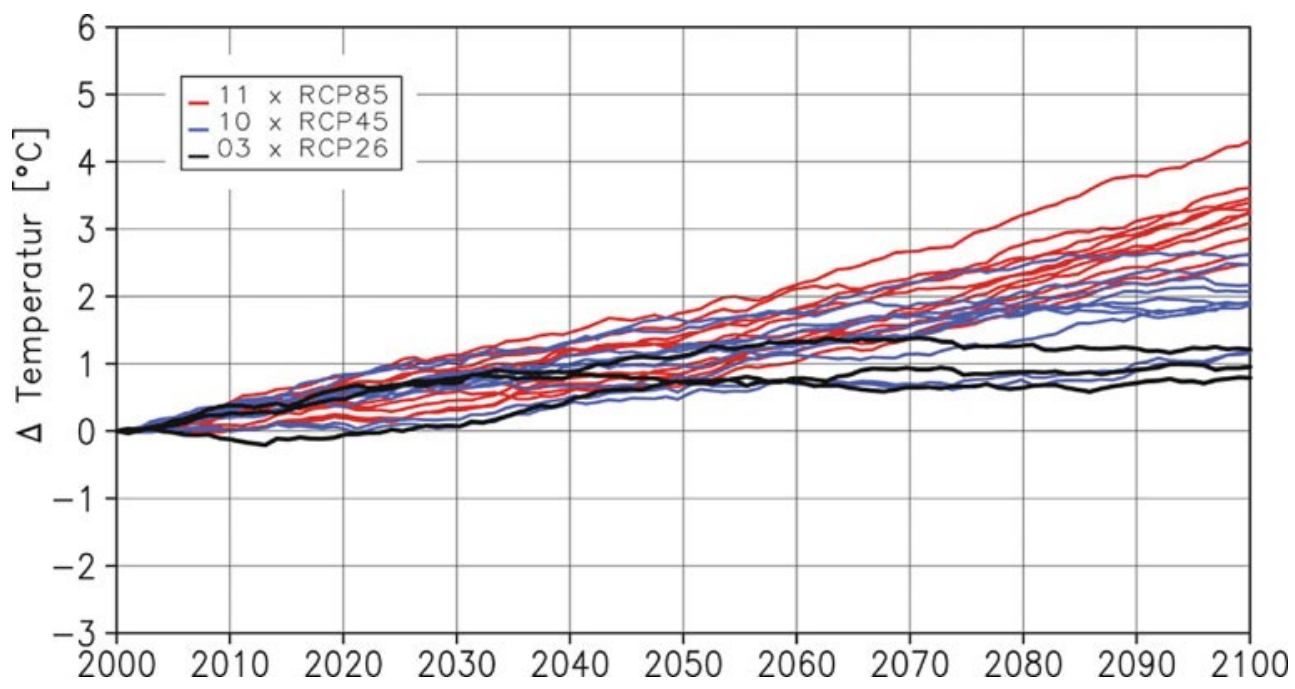

- Abb. 2.6 Projizierte Änderungen der jährlichen bodennahen Lufttemperatur ( 2 m über Grund) im Vergleich zu 1971-2000 als gleitendes 30-Jahres-Mittel, abgebildet jeweils auf das 30. Jahr für das Gebietsmittel der Metropolregion Hamburg, EURO-CORDEX-Simulationen (Jacob et al. 2014) verschiedener Global-/ Regionalmodell-Kombinationen auf Basis von RCP8.5 (rot), RCP4.5 (blau) und RCP2.6 (schwarz). (Abb. nach Rechid et al. 2014a) lich aus, im Frühjahr ist die Erwärmung im Vergleich geringer (ohne Abb.). Im RCP2.6 kann zum Ende des Jahrhunderts eine Stabilisierung der Temperaturänderung im Jahresmittel um rund $1{ }^{\circ} \mathrm{C}$ erreicht werden. Insgesamt zeigt sich, dass zur Mitte des Jahrhunderts die Bandbreiten der projizierten Temperaturänderungen hauptsächlich durch die Verwendung unterschiedlicher Modelle und die simulierte interne Klimavariabilität bestimmt wird, zum Ende des Jahrhunderts werden sie zunehmend durch die Emissionsszenarien beeinflusst. Im Verlauf des Jahrhunderts unterscheiden sich die für das B1- und RCP2.6- sowie RCP4.5Szenario simulierten Temperaturen immer deutlicher von den Ergebnissen für die A1B- und A2- sowie RCP8.5-Szenarien. Das bedeutet, dass durch eine Verminderung der Treibhausgasemissionen und damit geringere Treibhausgaskonzentrationen in der Atmosphäre auch in der Metropolregion Hamburg deutlich geringere Temperaturänderungen zu erwarten sind.

Im Norddeutschen Klimaatlas (Meinke und Gerstner 2009) werden Spannbreiten der jeweiligen Änderungen bezogen auf 30-jährige Zeitfenster zwischen 2011 und 2100 durch Differenzen zur Referenzperiode 1961-1990 ausgedrückt und sind somit nicht direkt mit den oben beschriebenen Auswertungen vergleichbar. Dennoch ist davon auszugehen, dass sich bestimmte Aussagen in beiden Auswertungen gleichermaßen wiederfinden, sofern es sich um robuste Trends handelt. Entsprechend den Auswertungen im Norddeutschen Klimaatlas scheint in der Metropolregion Hamburg und in Norddeutschland bis Mitte des 21. Jahrhunderts (2036-2065) eine Erwärmung zwischen etwa 1 und $3{ }^{\circ} \mathrm{C}$ plausibel. Bis Ende des 21. Jahrhunderts (2071-2100) kann sich diese Region um etwa $1-5^{\circ} \mathrm{C}$ erwärmen. Die Minima werden jeweils für Szenarien mit moderaten Treibhausgasemissionen projiziert (RCP2.6), während die Maxima dieser Spannbreiten für Szenarien mit hohen künftigen Treibhausgasemissionen abgebildet werden (RCP8.5). Somit werden die oben vorgestellten Auswertungen bestätigt und eingeordnet. Sowohl bis Mitte des Jahrhunderts als auch bis Ende des Jahrhunderts zeichnet sich den Auswertungen des Norddeutschen Klimaatlas zufolge die schwächste Erwärmung im Frühjahr ab. Bis Mitte des Jahrhunderts (2036-2065) können die mittleren Frühjahrstemperaturen je nach künftigem Treibhausgasausstoß nahezu unverändert bleiben bzw. bis etwa $3{ }^{\circ} \mathrm{C}$ ansteigen. Bis Ende des
Jahrhunderts (2071-2100) sind Erwärmungen zwischen 0,7 und $4,5^{\circ} \mathrm{C}$ im Frühjahr plausibel. Mit den stärksten jahreszeitlichen Erwärmungen ist im Sommer und im Herbst zu rechnen. Bis Ende des 21. Jahrhunderts können in dieser Jahreszeit die durchschnittlichen Temperaturen in der Hamburger Metropolregion und Norddeutschland um bis zu etwa $6{ }^{\circ} \mathrm{C}$ zunehmen. Gleichzeitig weisen diese Jahreszeiten auch die größten Spannbreiten plausibler Änderungen auf.

Die Verteilungen der täglichen Temperaturwerte in Rechid et al. (2014a) veranschaulichen, wie häufig welche Tagestemperaturen im heutigen und zukünftigen Klima auftreten. Eine markante Veränderung, die übereinstimmend in den Simulationen und Projektionszeiträumen von Rechid et al. (2014a) und BACC II Author Team (2015) auftritt, ist, dass im Winter die Anzahl sehr kalter und kalter Tage sehr viel stärker abnimmt als die Anzahl der milden Tage zunimmt. Das zeigt sich besonders deutlich bei der Auswertung der Perzentiländerungen. Die Verteilung der simulierten Tagesmitteltemperaturen im Winter wird danach im Zukunftsklima etwas schmaler. Auch die Auswertungen der thermischen Klimaindizes zeigen, dass niedrige Schwellenwerte deutlich seltener unterschritten werden, also Eis- und Frosttage seltener auftreten (Meinke et al. 2011; Rechid et al. 2014a). So zeigen auch die Auswertungen des Norddeutschen Klimaatlas, dass je nach Treibhausgasszenario Frosttage in der Hamburger Metropolregion gegen Ende des Jahrhunderts auf wenige Wochen beschränkt sein könnten und Tage, an denen das Temperaturmaximum $0{ }^{\circ} \mathrm{C}$ nicht überschreitet (Eistage), möglicherweise gar nicht mehr auftreten (Norddeutscher Klimaatlas (Stand Oktober 2015), Meinke und Gerstner 2009; Meinke et al. 2011). Die Anzahl der Tage mit Temperaturen von $>5{ }^{\circ} \mathrm{C}$ nimmt deutlich zu, was auf eine Verlängerung der thermischen Vegetationsperiode hindeutet. So könnte sich der thermische Vegetationsbeginn bis Ende des Jahrhunderts in der Hamburger Metropolregion um etwa 2-10 Wochen nach vorne verlagern und somit im Januar oder Februar liegen (vgl. Norddeutscher Klimaatlas; Meinke und Gerstner 2009; Meinke et al. 2011). Nach Rechid et al. (2014a) nehmen im B1-Szenario die Tageswerte der Temperatur im Sommer zur Mitte und zum Ende des Jahrhunderts gleichmäßig zu, d. h., die Häufigkeitsverteilung verschiebt sich weitgehend gleichmäßig zu höheren 
- Abb. 2.7 Projizierte relative Änderungen der Niederschlagsmenge im Winter im Vergleich zu 1971-2000 als gleitendes 30-Jahres-Mittel, abgebildet jeweils auf dem 30. Jahr für das Gebietsmittel der Metropolregion Hamburg, EURO-CORDEX-Simulationen (Jacob et al. 2014) verschiedener Global-/ Regionalmodell-Kombinationen auf der Basis von RCP8.5 (rot), RCP4.5 (blau) und RCP2.6 (schwarz). (Abb. nach Rechid et al. 2014a)

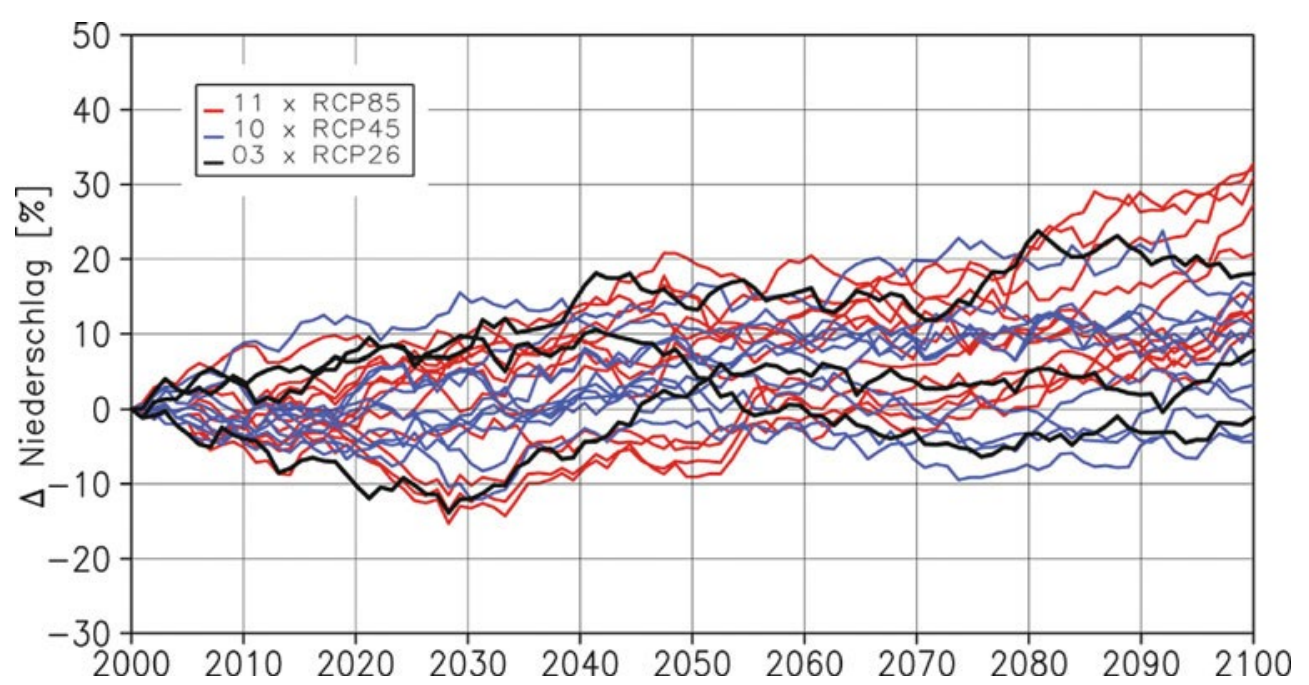

Temperaturen. Für das A1B- und das A2-Szenario zeigt sich dagegen zur Mitte und noch ausgeprägter zum Ende des Jahrhunderts ein etwas stärkerer Anstieg der höheren Perzentile, also der warmen bis heißen Tage, im Vergleich zu den niedrigeren Perzentilen, also den vergleichsweise kühlen Tagen. Dadurch wird die Verteilung auftretender Temperaturwerte etwas breiter, d. h., die Temperaturen können im Sommer stärker schwanken und warme und heiße Tage sehr viel häufiger auftreten. Das zeigen auch die Auswertungen in Rechid et al. (2014a) zu den Klimaindizes. Eine deutlich höhere Anzahl an Tagen erreicht oder überschreitet die $25^{\circ} \mathrm{C}$-Schwelle (Sommertage), und auch heiße Tage mit einer Maximumtemperatur von $30^{\circ} \mathrm{C}$ und mehr treten häufiger auf. Auch die Auswertungen im Norddeutschen Klimaatlas weisen in diese Richtung. So könnte sich bei starkem Anstieg künftiger Treibhausgasemissionen die Anzahl der Sommertage bis Ende des 21. Jahrhunderts in der Metropolregion Hamburg etwa verdoppeln, während heiße Tage dann ungefähr so häufig auftreten könnten wie gegenwärtig die Sommertage. Auch tropische Nächte $\left(\mathrm{T}_{\min } \geq 20^{\circ} \mathrm{C}\right)$ könnten in Zukunft bis zu viermal in jedem Jahr auftreten (vgl. Rechid et al. 2014a). Die Auswertungen im Norddeutschen Klimaatlas lassen bei starkem künftigem Treibhausgasausstoß (RCP8.5) Ende des Jahrhunderts sogar Jahre mit bis zu 34 tropischen Nächten plausibel erscheinen (Meinke und Gerstner 2009; Meinke et al. 2011). Werden Treibhausgasemissionen künftig jedoch nennenswert reduziert, kann die Häufigkeitszunahme von Sommertagen und heißen Tage bis Ende des Jahrhunderts auf wenige Tage begrenzt und die Häufigkeit tropischer Nächte sogar unverändert bleiben (Norddeutscher Klimaatlas (Stand Oktober 2015); Meinke und Gerstner 2009; Meinke et al. 2011).

Entsprechend den hier dokumentierten Ergebnisse liegt Konsens hinsichtlich einer Erwärmung im Laufe des 21. Jahrhunderts in der Metropolregion Hamburg vor. Bei weltweit erfolgreicher Umsetzung von Klimaschutzmaßnahmen kann die Erwärmung in dieser Region im Vergleich zum heutigen Temperaturniveau auf $1{ }^{\circ} \mathrm{C}$ bis Ende des Jahrhunderts begrenzt werden. Gelingt dies jedoch nicht, ist künftig mit einer beschleunigten Erwärmung zu rechnen, die bis Ende des Jahrhunderts in der Hamburger Metropolregion bis etwa $+5^{\circ} \mathrm{C}$ erreichen kann. Zudem können sich die Temperaturextrema hin zu einem wärmeren Klima mit mehr heißen Tagen und weniger Frosttagen verschieben. Diese Aussagen bestätigen im Wesentlichen die dokumentierten Erkenntnisse des 1 . HKB. Es zeichnet sich jedoch ab, dass sich mit einer zunehmenden Zahl von regionalen Klimaprojektionen die Spannbreiten von plausiblen Änderungen für die MRH erweitern. Auch die im Vergleich zu anderen Jahreszeiten eher moderate Erwärmung im Frühjahr ist mit den Aussagen des 1. HKB konsistent.

\subsubsection{Niederschlag}

Projizierte Änderungen des Niederschlags für die Metropolregion Hamburg auf Basis der SRES-Szenarien (s. o.) wurden in Rechid et al. (2014a) konsistent ausgewertet. Dort nimmt der Jahresniederschlag ab der Zeitperiode 2015-2045 in allen Simulationen zu, zum Ende des Jahrhunderts mit Werten zwischen 5 und $20 \%$. Die Zeitreihen zeigen eine hohe interannuelle und dekadische Variabilität des Niederschlags, die im Winter größer ist als im Sommer. Zur Mitte des Jahrhunderts liegen im Sommer die Ergebnisse aller Simulationen innerhalb von ca. -10 bis $+9 \%$ und damit etwa innerhalb der natürlichen Schwankungsbreite. Zum Ende des Jahrhunderts liegt im Sommer die Spannbreite zwischen etwa -20 und $+5 \%$. Werden allerdings regionale Klimaprojektionen basierend auf Simulationen mit verschiedenen Globalmodellen betrachtet (z. B. BACC II Author Team 2015; Jacob et al. 2014), dann reicht in der Metropolregion Hamburg die Bandbreite der mittleren Niederschlagsänderungen im Sommer zum Ende des 21. Jahrhunderts von etwa -30 bis $+20 \%$. In - Abb. 2.7 sind die auf der Basis der EURO-CORDEX-EUR11-Simulationen projizierten prozentualen Änderungen des Niederschlags im Vergleich zum Zeitraum 1971-2000 für den Winter als gleitendes 30-Jahres-Mittel (jeweils abgebildet auf das 30. Jahr) für das Gebietsmittel der Metropolregion Hamburg dargestellt, in - Abb. 2.8 findet sich die entsprechende Darstellung für die Niederschlagsänderung im Sommer. Auch hier zeigt sich die hohe dekadische Variabilität, die auch in den 30-jährigen Mittelwerten deutlich zu sehen ist. Für den Winter zeigen fast alle Simulationen eine Niederschlagszunahme mit einer Bandbreite von leicht negativen Werten bis etwa $+30 \%$ zum Ende des Jahrhunderts im Vergleich zu 1971-2000. Im Sommer hingegen zeigen etwa gleich viele Simulationen eine Zunahme wie 


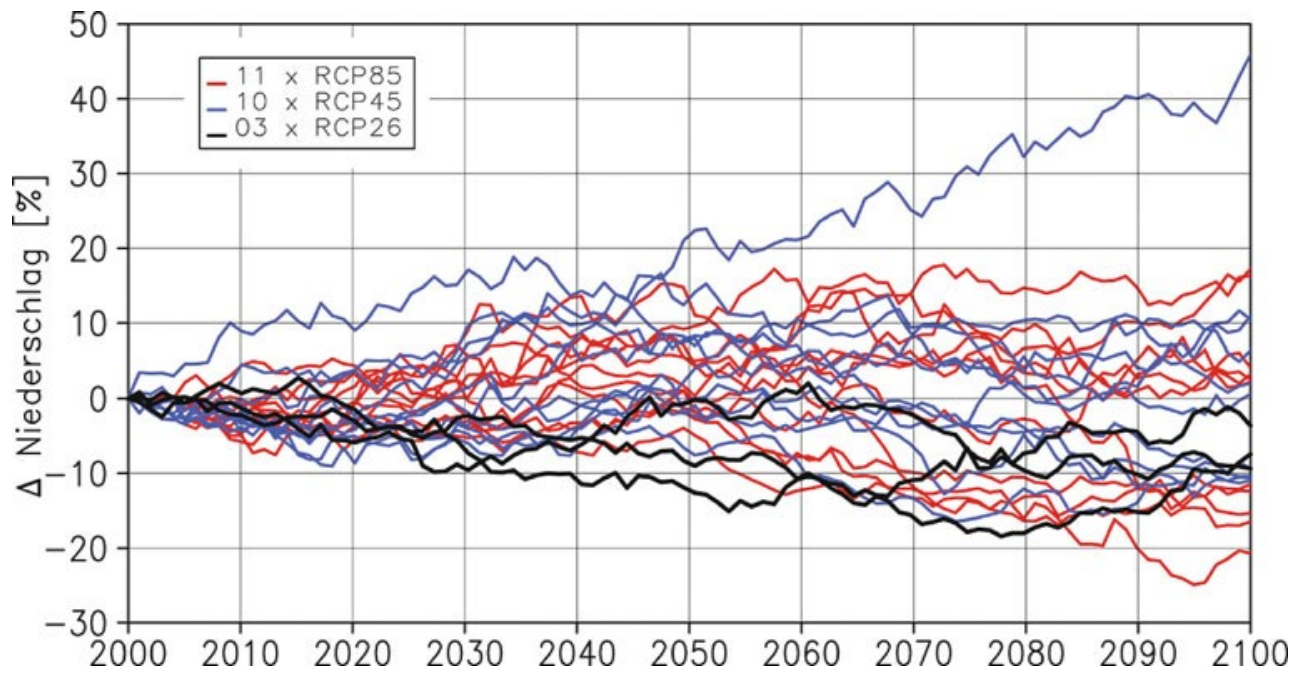

- Abb. 2.8 Projizierte relative Änderungen der Niederschlagsmenge im Sommer im Vergleich zu 1971-2000 als gleitendes 30-JahresMittel, abgebildet jeweils auf dem 30. Jahr für das Gebietsmittel der Metropolregion Hamburg, EUROCORDEX-Simulationen (Jacob et al. 2014) verschiedener Global-/ Regionalmodell-Kombinationen auf der Basis von RCP8.5 (rot), RCP4.5 (blau) und RCP2.6 (schwarz). (Abb. nach Rechid et al. 2014a)
Abnahme. Für eine einzelne Simulation wird bis etwa $+40 \%$ Niederschlag zum Ende des Jahrhunderts simuliert, sonst etwa zwischen -20 und $+20 \%$ (• Abb. 2.8).

Großräumig betrachtet verläuft im Sommer durch Deutschland im Ensemble-Mittel der Übergangsbereich von abnehmenden Niederschlägen in Südwesteuropa und zunehmenden Niederschlägen in Nordeuropa (Jacob et al. 2014).

Die Auswertungen im Norddeutschen Klimaatlas (Stand Oktober 2015, Meinke und Gerstner 2009) bestätigen im Wesentlichen die oben beschriebenen möglichen Niederschlagentwicklungen im 21. Jahrhundert: Auf Basis des gemischten Ensembles von regionalen SRES-Szenarien (A2, A1B, B2 und B1) und RCPSzenarien (CORDEX-EUR-44- und EUR-11-Simulationen) sind in der Metropolregion Hamburg bis Ende des 21. Jahrhunderts (2071-2100) im Vergleich zum Referenzzeitraum 1961-1990 jährliche Niederschlagszunahmen um bis zu etwa $30 \%$ plausibel, wobei auch eine leichte Abnahme der jährlichen Niederschlagsmenge von $8 \%$ bis Ende des Jahrhunderts nicht auszuschließen ist. Die größte Übereinstimmung der 123 regionalen Klimaszenarien hinsichtlich jahreszeitlicher Niederschlagsänderungen bis Ende des Jahrhunderts liegt im Winter mit Zunahmen von bis zu 41 \% vor. Im Sommer weisen viele regionale Projektionen auf Abnahmen hin, die bis zu $45 \%$ betragen können. Wie sich bereits bei den oben beschriebenen Ensemble-Auswertungen abzeichnet, ist die Entwicklung des Sommerniederschlags in der Hamburger Metropolregion jedoch unklar, da die Modellsimulationen Niederschlagsentwicklungen mit unterschiedlichen Vorzeichen projizieren. Demnach sind aus heutiger Sicht bis Ende des Jahrhunderts auch deutliche sommerliche Niederschlagszunahmen von bis $\mathrm{zu}$ $47 \%$ plausibel. Auch in den anderen Jahreszeiten weisen die Simulationen bzgl. der Niederschlagsentwicklung unterschiedliche Vorzeichen auf, sodass die künftige Niederschlagsentwicklung aus heutiger Sicht unklar bleibt. Maximale Änderungen werden aber auch beim Niederschlag für Szenarien mit hohen künftigen Treibhausgasemissionen projiziert (Norddeutscher Klimaatlas; Meinke und Gerstner 2009; Meinke et al. 2011).

Um räumlich differenzierte Aussagen zu projizierten Niederschlagsänderungen zu treffen, kann eine Analyse zur Robustheit der simulierten Änderungssignale z. B. nach Pfeifer et al. (2015) oder nach Meinke (2013) durchgeführt werden. Nach dieser Me- thode werden die Übereinstimmung der Modellergebnisse in der Richtung des Änderungssignals und die Signifikanz der Ergebnisse für jede Simulation untersucht und daraus eine Aussage zur Robustheit der projizierten Änderungen auf Basis jeweils eines konsistenten Modellensembles abgeleitet. Für den mittleren Sommerniederschlag kann danach für kein Gebiet in der Metropolregion Hamburg eine robuste Veränderung für die verschiedenen Szenarien und Modellensembles gezeigt werden. Für den mittleren Winterniederschlag werden auf Basis der EUROCORDEX-EUR-11-Simulationen für RCP8.5 robuste Zunahmen gegen Ende des 21. Jahrhunderts projiziert, auf Basis der ENSEMBLES-Simulationen für SRES A1B hingegen schon zur Mitte des 21. Jahrhunderts (Pfeifer et al. 2015).

Die Verteilung der Niederschlagswerte im Jahr und in den Jahreszeiten, die an Tagen mit mehr als $1 \mathrm{~mm}$ Niederschlag auftreten, wurden in Rechid et al. (2014a) auf Basis der REMO- und CLM-Simulationen ausgewertet. Dabei zeigt sich für den Winter in allen Simulationen eine generelle Zunahme aller Tagesniederschlagswerte und besonders zum Ende des Jahrhunderts mit einer Tendenz zur stärkeren Zunahme hoher Niederschlagsmengen. Auch auf Basis der ENSEMBLES-Projektionen konnte eine $\mathrm{Zu}$ nahme des 95. Perzentils der Tagesniederschläge gezeigt werden (Rudolf 2011). Im Sommer wird zum Ende des 21. Jahrhunderts in allen Simulationen eine Abnahme der Niederschlagsmengen an Tagen mit leichten bis mittleren Niederschlagsintensitäten projiziert. An Tagen mit hohen Niederschlagsmengen zeigt sich dagegen eine Zunahme der Niederschläge. Nach Auswertungen im Norddeutschen Klimaatlas (Stand Oktober 2015) ist die Häufigkeitsentwicklung von Tagen mit mindestens $1 \mathrm{~mm}$ Niederschlag unklar, da die jahreszeitlichen und jährlichen Trends in den einzelnen Simulationen unterschiedliche Vorzeichen aufweisen. Bei der Häufigkeitsentwicklung von Niederschlagstagen mit mehr als 10 bzw. $20 \mathrm{~mm}$ Niederschlag pro Tag stimmen die Simulationen jedoch in allen Jahreszeiten außer im Sommer in einer möglichen Zunahme überein.

Entsprechend den hier dokumentierten Ergebnissen bzgl. der möglichen zukünftigen Niederschlagsentwicklung in der Metropolregion Hamburg lässt sich dahingehend Konsens ableiten, dass die plausiblen Trends große Spannbreiten und unterschiedliche Vorzeichen aufweisen. Vor allem im Sommer 
und im Winter muss künftig mit deutlich veränderten Niederschlagsmengen gerechnet werden. Bei geringem Treibhausgasausstoß sind auch beim Niederschlag geringere Änderungen zu erwarten. Insgesamt kann die Häufigkeit von Tagen mit hohen Niederschlagsmengen in der Hamburger Metropolregion bis Ende des Jahrhunderts zunehmen. Hinsichtlich der Aussagen im 1. HKB zeigt sich vor allem für die künftige sommerliche Niederschlagsentwicklung ein Unterschied, da auf der Grundlage weiterer inzwischen ausgewerteter Klimaprojektionen bis Ende des Jahrhunderts auch eine deutliche Niederschlagszunahme in der Hamburger Metropolregion plausibel ist.

\subsubsection{Atmosphärische Zirkulation und Wind}

Die anthropogen verursachten projizierten Änderungen der Strahlungsbilanz, des Energiehaushalts und des Wasserkreislaufs beeinflussen die Massen- und Druckverteilung in der Atmosphäre und damit die atmosphärische Zirkulation. Die atmosphärischen Zirkulationsverhältnisse unterliegen zugleich natürlichen periodischen Schwankungen und in Wechselwirkung mit dem Ozean auch auf vergleichsweise langen Zeitskalen von mehreren Jahren und Jahrzehnten. Deshalb ist eine Ableitung von langfristigen robusten Trends für die Zukunft bislang kaum möglich, da die projizierten Änderungen meist in der Größenordnung der natürlichen Schwankungen liegen.

Diese natürlichen Schwankungen (interne Variabilität) des Klimas in Mitteleuropa und der Metropolregion Hamburg werden maßgeblich durch die Zirkulationsverhältnisse über dem Nordatlantik - die „Nordatlantische Oszillation“ (NAO) geprägt $(\triangle$ Abschn. 2.3.1). Die NAO beeinflusst die Bewegung der planetaren Wellen und die Zugbahnen von Tiefdruckgebieten über den Nordatlantik. Studien zu möglichen Änderungen der NAO auf der Basis von unterschiedlichen Klimaprojektionen mit verschiedenen globalen Klimamodellen führen zu unterschiedlichen und oft widersprüchlichen Ergebnissen (IPCC 2013; BACC II Author Team 2015). Zudem gibt es verschiedene Methoden zur Bestimmung der NAO, die ebenfalls zu unterschiedlichen Ergebnissen führen können (z. B. Ulbrich et al. 2013). Abschn. 2.3.1 hat gezeigt, dass sich in der Vergangenheit Zugbahnen von Tiefdruckgebieten über dem Nordatlantik nach Nordosten verlagert haben. Ob diese Veränderungen auf natürlichen Schwankungen beruhen oder durch den Einfluss des Menschen auf das Klima verursacht wurden und wie sich die Zirkulationsverhältnisse in der Region in Zukunft verändern können, ist weiterhin unklar.

Die Simulation zukünftig möglicher Änderungen der Windgeschwindigkeiten hängen sehr stark davon ab, wie die großskalige atmosphärische Zirkulation in den globalen Klimamodellen für die Zukunft abgebildet wird und in welchem Modus der periodischen Schwankungen sie sich jeweils befinden. Dabei gehen die Ergebnisse unterschiedlicher Modelle deutlich auseinander, sodass keine generellen Aussagen zu Veränderungen der Windgeschwindigkeiten getroffen werden können (BACCII Author Team 2015).

Die klimatischen Windverhältnisse können auch durch vorherrschende Windrichtungen charakterisiert werden. Besonders die Windrichtungen bei hohen Windgeschwindigkeiten beeinflussen neben der Windstärke die Sturmflutgefährdung in der deutschen Bucht. De Winter et al. (2013) z. B. untersuchen die projizierten Änderungen der Windrichtungen der jährlichen Windmaxima auf der Basis von 12 Globalmodellen des CMIP5 für das RCP4.5 und RCP8.5. Zunächst zeigt auch diese Studie, dass die Windgeschwindigkeiten großen Schwankungen unterliegen und keine Trends z. B. für die jährlichen Maxima der Windgeschwindigkeit abgeleitet werden können. Auch die Schwankungen der Windrichtungen sind groß; allerdings weisen hier die Ergebnisse darauf hin, dass die Ereignisse mit den jährlich maximalen Windgeschwindigkeiten im Verlauf des 21. Jahrhunderts häufiger aus südwestlicher und westlicher Richtung kommen können. Für Winde aus nordwestlicher und nordnordwestlicher Richtung hingegen, die für die Entwicklung von Sturmfluten entscheidend sind, wurden keine Änderungen der Windrichtung projiziert. Die Ergebnisse dieser Studie bestätigen Ergebnisse auf der Basis von globalen Modellsimulationen des CMIP3 (de Winter et al. 2013). Gaslikova et al. (2013) untersuchen das Windklima der deutschen Bucht auf der Basis von regionalen Klimamodelldaten eines dynamischen Regionalmodells in Kombination mit einem Globalmodell mit je zwei Realisierungen für die beiden SRES-Szenarien B1 und A1B. Auch diese Studie zeigt für die Deutsche Bucht im Verlauf des 21. Jahrhunderts eine Tendenz zur Zunahme starker Winde $\left(>17,2 \mathrm{~ms}^{-1}\right.$ ) aus westlicher Richtung, auch hier überlagert durch große multidekadische Schwankungen.

Insgesamt zeigt sich, dass es zur Interpretation von Klimaprojektionen auf der Grundlage verschiedener Studien wichtig ist, die jeweils verwendeten Daten, Methoden und zeitlichen Referenzperioden zu beachten. Das gilt insbesondere auch für die Projektionen von Windgeschwindigkeiten und der Häufigkeit von Sturmereignissen. Feser et al. (2015) geben einen systematischen Überblick über Studien zur Entwicklung der Sturmaktivitäten im Nordatlantik und Nordwesteuropa, die sie nach verwendeten Datensätzen, Analysemethoden und jeweils betrachteten geographischen Regionen und räumlichen Zeitperioden klassifizieren. Aufgrund der großen zeitlichen Variabilität der Sturmaktivitäten in Nordwesteuropa sind die Ergebnisse sehr stark von der Länge der untersuchten Zeitperiode abhängig. Die Projektionen für mögliche zukünftige Entwicklungen weisen zudem eine sehr starke Abhängigkeit von dem zugrunde liegenden Modellensemble auf. Nach Feser et al. (2015) stimmen viele Studien darin überein, dass unter zukünftigen Klimabedingungen die Intensität von Sturmereignissen in allen dort untersuchten geographischen Regionen und damit auch der Metropolregion Hamburg zunimmt. Outten und Esau (2013) hingegen erhalten auf der Basis von Klimaprojektionen für das A1B-Szenario des ENSEMBLES-Projekts keine wesentliche Änderung in der Statistik hoher Windgeschwindigkeiten in Bodennähe. Laut Feser et al. (2015) deutet die Mehrzahl der Artikel auf eine künftige Häufigkeitszunahme von Sturmereignissen über der Nordsee hin. Für die Ostsee konnte dies jedoch nicht festgestellt werden. Entsprechend heterogene Ergebnisse zeigen auch die Auswertungen des Norddeutschen Klimaatlas (Stand Oktober 2015, Meinke und Gerstner 2009; Meinke et al. 2011) bzgl. möglicher künftiger Änderungen der Windgeschwindigkeiten bei Sturmereignissen und deren Häufigkeit in der Metropolregion Hamburg. Bis Ende des Jahrhunderts (2071-2100) sind im Vergleich zur Referenzperiode 1961-1990 Änderungen der maximalen Windgeschwindigkeiten 
(maximaler Betrag des Windvektors in $10 \mathrm{~m}$ Höhe) von -4 bis $+4 \%$ plausibel. Auch innerhalb der Jahreszeiten weisen die regionalen Klimaprojektionen unterschiedliche Vorzeichen in den Trends künftiger Sturmintensitäten auf. Die größten Spannbreiten in der Änderung maximaler Windstärken treten in der Metropolregion Hamburg im Winter auf: Sie liegen zwischen -8 und $+10 \%$. Bei der Häufigkeit von Sturmereignissen zeigen die zugrunde gelegten Klimaprojektionen eine Spannweite von -8 bis +14 Sturmtagen (max. Windgeschwindigkeit überschreitet $8 \mathrm{Bft}$ ), wobei die höchste Zunahme im Winter zu erwarten ist.

Insgesamt können zur potenziellen künftigen Entwicklung von Sturmereignissen in der Metropolregion Hamburg keine robusten Aussagen getroffen werden. Bis Ende des 21. Jahrhunderts erscheint sowohl eine Zunahme als auch eine Abnahme der Windgeschwindigkeiten bei Sturmereignissen und ihrer Häufigkeit in der Metropolregion Hamburg möglich.

\subsection{Zusammenfassung und Ausblick}

Größtenteils werden die im 1. Klimabericht für die Metropolregion Hamburg getroffenen Aussagen zum Klima und seinen bisherigen sowie künftig möglichen Entwicklungen in der Metropolregion durch neue Veröffentlichungen seit 2009 bestätigt. Entsprechend den hier dokumentierten Ergebnissen besteht insbesondere Konsens hinsichtlich einer bereits stattfindenden Erwärmung, die sich im Laufe des 21. Jahrhunderts in der MRH weiter fortsetzen wird. Bei weltweit erfolgreicher Umsetzung von Klimaschutzmaßnahmen kann die Erwärmung in dieser Region im Vergleich zum heutigen Temperaturniveau auf $1^{\circ} \mathrm{C}$ bis Ende des Jahrhunderts begrenzt werden. Gelingt dies jedoch nicht, ist künftig mit einer beschleunigten Erwärmung zu rechnen, die bis Ende des Jahrhunderts in der MRH etwa $+5^{\circ} \mathrm{C}$ erreichen kann.

Im Vergleich zum 1. HKB wirken sich hinsichtlich der bisherigen Entwicklung bei einigen meteorologischen Größen der zwischenzeitlich fortgeschrittene Klimawandel, fortlaufende natürliche Schwankungen und ggf. weitere anthropogene Einflüsse auf die Statistiken aus. Hinsichtlich der Aussagen zu möglichen zukünftigen Klimaänderungen haben zusätzliche regionale Klimaprojektionen dazu geführt, dass größere Spannbreiten möglicher zukünftiger Änderungen bestimmter Klimaelemente abgebildet werden. Dies betrifft vor allem mögliche zukünftige Änderungen des Niederschlags und der Windverhältnisse.

Insgesamt zeigt sich, dass die Verwendung möglichst vieler Global-/Regionalmodell-Kombinationen wichtig ist, um das breite Spektrum der Ergebnisse verschiedener Modelle in den Klimaprojektionen abzubilden. Zur Interpretation der Ergebnisse verschiedener Studien ist es wichtig, die jeweils verwendeten Daten, Methoden sowie räumlichen Skalen und zeitlichen Referenzperioden zu beachten. Im Verlauf des 21. Jahrhunderts unterscheiden sich die für das B1- und RCP2.6- sowie das RCP4.5-Szenario simulierten Temperaturzunahmen immer deutlicher von den Ergebnissen für die A1B- und A2- sowie die RCP8.5-Szenarien. Das bedeutet, dass durch eine Verminderung der Treibhausgasemissionen in der ersten Hälfte des 21. Jahrhunderts und damit geringere Treibhausgaskonzentrationen in der Atmosphäre zum Ende des 21. Jahrhunderts deutlich geringere Klimaänderungen zu erwarten sind.
Im RCP2.6 kann zum Ende des Jahrhunderts gegenüber 19712000 eine Stabilisierung der Temperaturänderung im Jahresmittel auf $1{ }^{\circ} \mathrm{C}$ und damit auf etwa $2{ }^{\circ} \mathrm{C}$ gegenüber dem vorindustriellen Niveau erreicht werden. Die simulierten zeitlichen Entwicklungen des Niederschlags zeigen eine hohe dekadische Variabilität, die auch in den 30-jährigen Mittelwerten deutlich zu sehen ist. Für den Winter projizieren fast alle Simulationen eine Niederschlagszunahme ab der Mitte des Jahrhunderts. Für den Sommer hingegen können keine robusten Niederschlagsänderungen aus den Simulationen abgeleitet werden.

Bisherige Klimaentwicklungen und deren Konsistenz mit regionalen Klimaprojektionen sollen alle 5-10 Jahre hinsichtlich der jüngsten Entwicklungen aktualisiert werden. Die Analyse von Beobachtungsdaten, Rasterdatensätzen und Reanalysen über das Jahr 2010 hinaus bis 2015 zeigen übereinstimmend, dass sich die Erwärmung und die winterliche Niederschlagszunahme in der Metropolregion weiter fortgesetzt haben.

Derzeit werden in dem vom BMBF geförderten nationalen Verbundprojekt ReKliEs-De die EURO-CORDEX-Simulationen durch weitere dynamische und auch statistische Regionalisierungen auf der Basis weiterer globaler Projektionen ergänzt, um dem neuesten Wissensstand entsprechende regionale Klimaprojektionen auf der Grundlage großer Multi-Modell-Ensembles für Deutschland zu erstellen. Dabei soll auch die Stabilität von Ergebnissen auf Grundlage unterschiedlicher Ensemblegrößen untersucht werden. Ziel des Projekts ist die Bereitstellung verlässlicher Informationen über die Bandbreite und Extreme der zukünftigen Klimaentwicklung für das Gebiet von Deutschland einschließlich der Einzugsgebiete der großen nach Deutschland entwässernden Flüsse. Hierzu gehört insbesondere auch die nutzerorientierte Aufbereitung der wissenschaftlichen Ergebnisse für die Verwendung in der Klimafolgenforschung und der Politikberatung.

\section{Literatur}

Alexandersson $\mathrm{H}$, Tuomenvirta $\mathrm{H}$, Schmith T, Iden $\mathrm{K}$ (2000) Trends of storms in NW Europe derived from an updated pressure data set. Clim Res 14:71-73 American Meteorological Society (2012) Glossary of meteorology. Webseite der AMS. Zugegriffen: 17. März 2017

Author Team BACC II (2015) Second assessment of climate change for the baltic sea Basin. Springer, Heidelberg, New York, Dordrecht, London

Bhend J, von Storch H (2008) Consistency of observed winter precipitation trends in northern Europe with regional climate change projections. Clim Dynam 31:17-28

Bhend J, von Storch H (2009) Is greenhouse gas warming a plausible explanation for the observed warming in the Baltic Sea catchment area? Boreal Environ Res 14:81-88

Booß A, Lefebvre C, Löpmeier F-J, Müller-Westermeier G, Pietzsch S, Riecke W, Schmitt H-H (2011) Die Witterung in Deutschland 2010. Klimastatusbericht 2010. Deutscher Wetterdienst, Offenbach am Main

BSH (2013) Sturmflut vom 07.12.2013 (Webseiten des BSH)

Bülow K, Ganske A, Hüttl-Kabus S, Klein B, Klein H, Löwe P, Möller J, Schade N, Tinz B, Heinrich H, Rosenhagen G (2014) Klimabedingte Auswirkungen auf Schifffahrt, Küsten und Meeresnutzung in der Nordseeregion. Schlussbericht KLIWAS-Projekt 3.01. KLIWAS-35/2014

Casty C, Raible CC, Stocker TF, Wanner H, Luterbacher J (2007) A European pattern climatology 1766-2000. Clim Dyn 29:791-805

Chmielewski F-M (2011) Der Einfluss des Klimawandels auf den Wirtschaftssektor Landwirtschaft. In: von Storch H, Claussen M (Hrsg) Klimabericht für die Metropolregion Hamburg. Springer, Berlin, Heidelberg, S 211-230 
Christensen JH, Carter T, Giorgi F (2002) PRUDENCE employs new methods to assess european climate change. Eos (Washington DC) 83:147

Daschkeit A (2011) Das Klima der Region und mögliche Entwicklungen in der Zukunft bis 2100. In: von Storch H, Claussen M (Hrsg) Klimabericht für die Metropolregion Hamburg. Springer, Berlin, Heidelberg, S 61-90

Deutschländer T, Friedrich K, Haeseler S, Lefebvre C (2013) Orkantief XAVER über Nordeuropa vom 5. bis 7. Dezember 2013 (Webseiten des DWD)

DWD (2015) Wetterlexikon, Webseiten des DWD. Zuletzt zugegriffen am 30.10.2015

De Winter RC, Sterl A, Ruessink BG (2013) Wind extremes in the North Sea basin under climate change: an ensemble study of 12 CMIP5 GCMs. J Geophys Res 118:1601-1612

Feser F, Barcikowska M, Krueger O, Schenk F, Weisse R, Xia L (2015) Storminess over the North Atlantic and northwestern Europe - A review. Q J R Meteorol Soc 141:350-382

Fuss S, Canadell JG, Peters GP, Tavoni M, Andrew RM, Ciais P, Jackson RB, Jones CD, Kraxner F, Nakicenovic N, Le Quéré C, Raupach MR, Sharifi A, Smith P, Yamagata (2014) Betting on negative emissions. Nat Clim Chang 4:850-853

Gaslikova L, Grabemann I, Groll N (2013) Changes in North Sea storm surge conditions for four transient future climate realizations. Nat Hazards 66:1501-1518

Geyer B (2014) High resolution atmospheric reconstruction for Europe 19482012: coastDat2. Earth Syst Sci Data 6:147-164

Giorgi F, Jones C, Asrar G (2009) Addressing climate information needs at the regional level: the CORDEX framework. WMO Bull 58:175-183

Haeseler S, Lefebvre C (2013) Orkantief CHRISTIAN am 28. Oktober 2013. Zugegriffen: 17. März 2017 (Webseite des DWD)

Haeseler S, Lefebvre C, Friedrich A (2015) Unwetter mit Tornados richten am 5. Mai 2015 schwere Schäden in Norddeutschland an. Zugegriffen: 17. März 2017 (Webseite des DWD)

Hoffmann P (2009) Modifikation von Starkniederschlägen durch urbane Gebiete. Fachbereich Geowissenschaften Universität Hamburg, Diplomarbeit

Hollweg HD, Böhm U, Fast I, Hennemuth B, Keuler K, Keup-Thiel E, Lautenschlager M, Legutke S, Radtke K, Rockel B, Schubert M, Will A, Woldt M, Wunram $C$ (2008) Ensemble simulations over europe with the regional climate model CLM forced with IPCC AR4 global scenarios. M \& D technical report 3.

Hurrel JW, Kushnir Y, Ottersen G, Visbeck M (2003) An overview of the North Atlantic oscillation. In: Hurrel JW, Kushnir Y, Ottersen G, Visbeck M (Hrsg) The North Atlantic oscillation: climatic significance and environmental impact. Geophyical Monograph series 134. American Geophysical Union, Washington DC, S 1-36

Imbery F, Friedrich K, Koppe-Schaller C, Rösner S, Bissolli P, Schreiber K-J (2015) Erste klimatologische Einschätzung der Hitzewelle 2015 (Webseiten des DWD)

IPCC (2013) Climate change 2013: the physical science basis. Contribution of working group I to the fifth assessment report of the intergovernmental panel on climate change. Cambridge University Press, New York, Cambridge

Jacob D, Göttel H, Kotlarski S, Lorenz P, Sieck K (2008) Klimaauswirkungen und Anpassung in Deutschland: Erstellung regionaler Klimaszenarien für Deutschland mit dem Klimamodell REMO. Forschungsbericht 20441138 Teil 2. UBA, Dessau

Jacob D, Bülow K, Kotova L, Moseley C, Petersen J, Rechid D (2012) Regionale Klimaprojektionen für Europa und Deutschland: Ensemble Simulationen für die Klimafolgenforschung. CSC Report 6. Climate Service Center, Hamburg

Jacob D, Petersen J, Eggert B, Alias A, Christensen OB, Bouwer LM, Braun A, Colette A, Déqué $M$, Georgievski G, Georgopoulou E, Gobiet $A$, Menut $L$, Nikulin G, Haensler A, Hempelmann N, Jones C, Keuler K, Kovats S, Kröner N, Kotlarski S, Kriegsmann A, Martin E, van Meijgaard E, Moseley C, Pfeifer S, Preuschmann S, Radermacher C, Radtke K, Rechid D, Rounsevell M, Samuelsson P, Somot S, Soussana J-F, Teichmann C, Valentini R, Vautard R, Weber B, Yiou P (2014) EURO-CORDEX: new high-resolution climate change projections for European impact research. Reg Environ Change 14:563-578

Kaas E, Li TS, Schmith T (1996) Statistical hindcast of wind climatology in the North Atlantic and Northwestern European region. Clim Res 7:97-110

Kalnay E, Kanamitsu M, Kistler R, Collins W, Deaven D, Gandin L, Iredell M, Saha S, White G, Woollen J, Zhu Y, Leetmaa A, Reynolds R, Chelliah M, Ebisuzaki W, Higgins W, Janowiak J, Mo KC, Ropelewski C, Wang J, Jenne R, Joseph D
(1996) The NCEP/NCAR 40-year reanalysis project. Bull Am Meteorol Soc 77:437-471

Kaspar F, Müller-Westermeier G, Penda E, Mächel H, Zimmermann K, KaiserWeiss A, Deutschländer T (2013) Monitoring of climate change in Germany - data, products and services of Germany's National Climate Data Centre. Adv Sci Res 10:99-106

Kistler R, Kalnay E, Collins W, Saha S, White G, Woollen J, Chelliah M, Ebiszusaki W, Kanamitsu M, Kousky V, van den Dool H, Jenne R, Fiorino M (2001) The NCEP-NCAR 50 year reanalysis. Bull Am Meteorol Soc 82:247-267

KLIMZUG-NORD Verbund (2014) Kursbuch Klimaanpassung. Handlungsoptionen für die Metropolregion Hamburg. TuTech, Hamburg. ISBN 9783941492660

Knutti R, Sedláček J (2013) Robustness and uncertainties in the new CMIP5 coordinated climate model projections. Nat Clim Chang 3:369-373

Kotlarski S, Keuler K, Christensen OB, Colette A, Déqué M, Gobiet A, Goergen K, Jacob D, Lüthi D, van Meijgaard E, Nikulin G, Schär C, Teichmann C, Vautard R, Warrach-Sagi K, Wulfmeyer V (2014) Regional climate modeling on European scales: a joint standard evaluation of the EURO-CORDEX RCM ensemble. Geosci Model Dev 7:1297-1333

Kreienkamp F, Spektat A, Enke W (2011) Ergebnisse regionaler Szenarienläufe für Deutschland mit der statistischen Methode WETTREG auf der Basis der SRES Szenarios A2 und B1 modelliert mit ECHAM5/MPI-OM. CSC Report 2. Climate Service Center,

Leiding T, Tinz B, Rosenhagen G, Lefebvre C, Haeseler S, Hagemann S, Bastigkeit I, Stein D, Schwenk P, Müller S, Outzen O, Herklotz K, Kinder F, Neumann T (2014) Meteorological and oceanographic conditions at the FINO platforms during the severe storms Christian and Xaver. DEWI-Magazin 44:16-25 (Webseite des DWD. Zugegriffen: 17.3.2017)

Linde M, Hoffmann P, Petersen J, Rechid D, Schlünzen KH, Schoetter R (2014a) Veränderungen des Klimas in der Region Elmshorn. In: Nehlsen E, Kunert L, Fröhle P, Knieling J (Hrsg) Wenn das Wasser von beiden Seiten kommt Bausteine eines Leitbildes zur Klimaanpassung für Elmshorn und Umland. Berichte aus den KLIMZUG-NORD Modellgebieten, Bd. 3. TuTech, Hamburg Linde M, Hoffmann P, Petersen J, Rechid D, Schlünzen KH, Schoetter R (2014b) Klimaänderungen. In: Schlünzen KH, Linde M (Hrsg) Wilhelmsburg im Klimawandel. Ist-Situation und mögliche Veränderungen. Berichte aus den KLIMZUG-NORD Modellgebieten, Bd. 4. TuTech, Hamburg

van der Linden P, Mitchell JFB (Hrsg) (2009) ENSEMBLES: climate change and its impacts: summary of research and results from the ENSEMBLES project. Met Office Hadley Centre,

Matulla C, Schoener W, Alexandersson H, von Storch H, Wang XL (2008) European storminess: Late $19^{\text {th }}$ century to present. Clim Dynam 31:125-130

Martinez G, Blobel D (2014) RADOST Abschlussbericht 156. Ecologic Institute, Berlin

Meehl GA, Covey C, Taylor KE, Delworth T, Stouffer RJ, Latif M, McAvaney B, Mitchell JFB (2007) THE WCRP CMIP3 multimodel dataset: a new era in climate change research. Bull Am Meteorol Soc 88:1383-1394

Meinke I (2013) Übereinstimmungskarten im Klimaatlas. REKLIM Newsletter Nr. 3 27-27 Oktober 2013

Meinke I: Bisherige Klimaentwicklung in Norddeutschland: Norddeutscher Klimamonitor aktualisiert. REKLIM Report Oktober 2017 (in print)

Meinke I, Gerstner E-M (2009) Digitaler Norddeutscher Klimaatlas informiert über möglichen künftigen Klimawandel. DMG Mitt 3/2009:17

Meinke I, Gerstner E-M, von Storch H, Marx A, Schipper H, Kottmeier C, Treffeisen R, Lemke P (2010) Regionaler Klimaatlas Deutschland der Helmholtz-Gemeinschaft informiert im Internet über möglichen künftigen Klimawandel. DMG Mitt 2/2010:5-7

Meinke I, Weisse R, von Storch H (2011) Regionale Klimaszenarien in der Praxis - Beispiel Norddeutschland. Helmholtz-Zentrum Geesthacht, Geesthacht

Meinke I, Maneke M, Riecke W, Tinz B (2014) Norddeutscher Klimamonitor Klimazustand und Klimaentwicklung in Norddeutschland innerhalb der letzten 60 Jahre (1951-2010). DMG Mitt 01/2014:2-11

Moseley C, Panferov O, Döring C, Dietrich J, Haberlandt U, Ebermann V, Rechid D, Beese F, Jacob D (2012) Klimaentwicklung und Klimaszenarien. In: Niedersächsisches Ministerium für Umwelt, Energie und Klimaschutz, Regierungskommission Klimaschutz (Hrsg) Empfehlung für eine niedersächsische Strategie zur Anpassung an die Folgen des Klimawandels 
Moss RH, Edmonds JA, Hibbard KA, Manning MR, Rose SK, van Vuuren DP, Carter TR, Emori S, Kainuma M, Kram T, Meehl GA, Mitchell JFB, Nakicenovic N Riahi K, Smith SJ, Stouffer RJ, Thomson AM, Weyant JP, Wilbanks TJ (2010) The next generation of scenarios for climate change research and assessment. Nature 463:747-756

Müller-Westermeier G, Lefebvre C, Nitsche H, Riecke W, Zimmermann K (2008) Die Witterung in Deutschland, Klimastatusbericht 2007 (Webseiten des DWD)

Nakicenovic N, Swart R (Hrsg) (2000) Emission scenarios. Cambridge University Press, Cambridge (UK)

Orlowsky B, Gerstengarbe FW, Werner PC (2008) A resampling scheme for regional climate simulations and its performance compared to a dynamical RCM. Theor Appl Climatol 92:209-223

Outten SD, Esau I (2013) Extreme winds over Europe in the ENSEMBLES regional climate models. Atmos Chem Phys 13:5163-5172

Pfeifer S, Bülow K, Gobiet A, Hänsler A, Mudelsee M, Otto J, Rechid D, Teichmann C, Jacob D (2015) Robustness of ensemble climate projections analyzed with climate signal maps: seasonal and extreme precipitation for Germany. Atmosphere (Basel) 6:677-698

Rechid D, Petersen J, Schoetter R, Jacob D (2014a) Klimaprojektionen für die Metropolregion Hamburg. Berichte aus den KLIMZUG-NORD Modellgebieten, Bd. 1. TuTech, Hamburg

Rechid D, Petersen J, Schoetter R, Jacob D (2014b) Klimaprojektionen für das Modellgebiet Lüneburger Heide. In: Urban B, Becker J, Mersch I, Meyer W, Rechid D, Rottgardt E (Hrsg) Klimawandel in der Lüneburger Heide Kulturlandschaften zukunftsfähig gestalten. Berichte aus den KLIMZUGNORD Modellgebieten, Bd. 6. TuTech, Hamburg

Rechid D, Petersen J, Schoetter R, Jacob D (2014c) Klimaprojektionen für das Biosphärenreservat Niedersächsische Elbtalaue. In: Prüter J, Keienburg T, Schreck C (Hrsg) Klimafolgenanpassung im Biosphärenreservat Niedersächsische Elbtalaue - Modellregion für nachhaltige Entwicklung. Berichte aus den KLIMZUG-NORD Modellgebieten, Bd. 5. TuTech, Hamburg

Rechid D, Petersen J, Jacob D (2014d) „Klimaprojektionen für die Metropolregion Hamburg. In: KLIMZUG-NORD Verbund (Hrsg) (2014) Kursbuch Klimaanpassung. Handlungsoptionen für die Metropolregion Hamburg. TuTech, Hamburg

Riecke W, Rosenhagen G (2010) Das Klima in Hamburg - Entwicklung des Klimas in Hamburg und der Metropolregion. Berichte des DWD 234. Selbstverlag des Deutschen Wetterdienstes, Offenbach

Rogelj J, Meinshausen M, Knutti R (2012) Global warming under old and new scenarios using IPCC climate sensitivity range estimates. Nat Clim Chang 2:248-253

Rosenhagen G (2008) Meteorologischer Hintergrund II: Zur Entwicklung der Sturmaktivität in Mittel und Westeuropa. Promet 34 (1/2). Selbstverlag des Deutschen Wetterdienstes, Offenbach

Rosenhagen G (2013) Das Klima der Metropolregion auf Grundlage meteorologischer Messungen und Beobachtungen. Klimabericht für die Metropolregion Hamburg. Internet-Update 2013 (Webseiten des Klimaberichts Hamburg)

Rosenhagen G, Schatzmann M (2011) Das Klima der Metropolregion auf Grundlage meteorologischer Messungen und Beobachtungen. In: von Storch $\mathrm{H}_{\text {, }}$ Claußen M (Hrsg) Klimabericht für die Metropolregion Hamburg. Springer, Berlin, Heidelberg, S 19-59

Rudolf E-M (2011) Untersuchung von Starkniederschlägen der ENSEMBLES Regionalmodelle in der Metropolregion Hamburg. Bachelorarbeit im Studiengang Meteorologie, Universität Hamburg

Schlünzen KH, Hoffmann P, Rosenhagen G, Riecke W (2009) Long term changes and regional differences in temperature and precipitation in the metropolitan area of Hamburg. J Clim 30:1121-1136

Schoetter R, Hoffmann P, Rechid D, Schlünzen KH (2012) Evaluation and bias correction of regional climate model results using model evaluation measures. J Appl Meteor Clim 51:1670-1684

Spekat A, Enke W, Kreienkamp F (2007) Neuentwicklung von regional hoch aufgelösten Wetterlagen für Deutschland und Bereitstellung regionaler Klimaszenarios auf der Basis von globalen Klimasimulationen mit dem Regionalisierungsmodell WETTREG auf der Basis von globalen Klimasimulationen mit ECHAM5/MPI-OM T63 L31 2010 bis 2100 für die SRES-Szenarios $B 1, A 1 B$ und A2. Endbericht. Umweltbundesamt, Dessau von Storch H, Feser F, Haeseler S, Lefebvre C, Stendel M (2014) A violent midlatitude storm in northern Germany and Denmark 28 October 2013. Explaining extreme events of 2013 from a climate perspective. Bull Am Meteorol Soc 95(Suppl 9):76-78

Taylor KE, Stouffer RJ, Meehl GA (2012) An overview of CMIP5 and the experiment design. Bull Am Meteorol Soc 93:485-498

Trusilova K, Riecke W (2015) Klimauntersuchung für die Metropolregion Hamburg zur Entwicklung verschiedener meteorologischer Parameter bis 2050. Berichte des Deutschen Wetterdienstes 247. Selbstverlag des DWD, Offenbach

Ulbrich U, Leckebusch GC, Grieger J, Schuster M, Akperov M, Bardin MY, Feng Y, Gulev S, Inatsu M, Keay K, Kew SF, Liberato MLR, Lionello P, Mokhov II, Neu U, Pinto JG, Raible CC, Reale M, Rudeva I, Simmonds I, Tilinina ND, Trigo IF, Ulbrich S, Wang XL, Wernli H, The IMILAST TEAM (2013) Are greenhouse gas signals of Northern Hemisphere winter extra-tropical cyclone activity dependent on the identification and tracking algorithm? Meteorol Z 22:61-68

Vautard R, Gobiet A, Jacob D, Belda $M$, Colette A, Déqué $M$, Fernández J, García-Díez M, Goergen K, Güttler I, Halenka T, Karacostas T, Katragkou E, Keuler K, Kotlarski S, Mayer S, Meijgaard E, Nikulin G, Patarčić M, Scinocca J, Sobolowski S, Suklitsch M, Teichmann C, Warrach-Sagi K, Wulfmeyer V, Yiou $P$ (2013) The simulation of European heat waves from an ensemble of regional climate models within the EURO-CORDEX project. Clim Dyn 41:2555-2575

Wagner S, Berg P, Schädler G, Kunstmann H (2013) High resolution regional climate model simulations for Germany: Part II - projected climate changes. Clim Dynam 40(1-2):415-427

WASA Group (1998) Changing waves and storms in the Northeast Atlantic? Bull Am Meteorol Soc 79(5):741-760

Weisse R, von Storch H, Feser F (2005) Northeast Atlantic and North Sea storminess as simulated by a regional climate model 1958-2001 and comparison with observations. J Clim 18:465-479

Weisse R, von Storch H, Callies U, Chrastansky A, Feser F, Grabemann I, Günther H, Winterfeldt J, Woth K, Pluess A, Stoye T, Tellkamp J (2009) Regional meteorological-marine reanalyses and climate change projections. Bull Am Meteorol Soc 90:849-860

Willmott CJ, Matsuura K (2012) Terrestrial air temperature: 1900-2010. Gridded Monthly Time Series (V 3.01). University of Delaware, Delaware

Open Access Dieses Kapitel wird unter der Creative Commons Namensnennung - Nicht kommerziell 4.0 International Lizenz (http://creativecommons.org/licenses/by-nc/4.0/deed.de) veröffentlicht, welche die nicht-kommerzielle Nutzung, Vervielfältigung, Bearbeitung, Verbreitung und Wiedergabe in jeglichem Medium und Format erlaubt, sofern Sie den/die ursprünglichen Autor(en) und die Quelle ordnungsgemäß nennen, einen Link zur Creative Commons Lizenz beifügen und angeben, ob Änderungen vorgenommen wurden.

Die in diesem Kapitel enthaltenen Bilder und sonstiges Drittmaterial unterliegen ebenfalls der genannten Creative Commons Lizenz, sofern sich aus der Abbildungslegende nichts anderes ergibt. Sofern das betreffende Material nicht unter der genannten Creative Commons Lizenz steht und die betreffende Handlung nicht nach gesetzlichen Vorschriften erlaubt ist, ist auch für die oben aufgeführten nicht-kommerziellen Weiterverwendungen des Materials die Einwilligung des jeweiligen Rechteinhabers einzuholen. 